\title{
Double power-law spectra of energetic electrons in the Earth magnetotail
}

\author{
A. V. Artemyev ${ }^{1,2}$, M. Hoshino ${ }^{3}$, V. N. Lutsenko ${ }^{1}$, A. A. Petrukovich ${ }^{1}$, S. Imada ${ }^{4}$, and L. M. Zelenyi ${ }^{1}$ \\ ${ }^{1}$ Space Research Institute, RAS, Moscow, Russia \\ ${ }^{2}$ LPC2E/CNRS - University of Orleans, UMR7328, Orleans, France \\ ${ }^{3}$ Department of Earth and Planetary Science, University of Tokyo, Tokyo, Japan \\ ${ }^{4}$ Institute of Space and Astronautical Science, Japan Aerospace Exploration Agency, Sagamihara, Japan
}

Correspondence to: A. V. Artemyev (ante0226@yandex.ru)

Received: 5 July 2012 - Revised: 1 November 2012 - Accepted: 17 December 2012 - Published: 18 January 2013

\begin{abstract}
In this paper, we consider electron acceleration in the vicinity of X-line and corresponding formation of energy spectra. We develop an analytical model including the effect of the electron trapping by electrostatic fields and surfing acceleration. Speiser, Fermi and betatron mechanisms of acceleration are also taken into account. Analytical estimates are verified by the numerical integration of electron trajectories. The surfing mechanism and adiabatic heating are responsible for the formation of the double power-law spectrum in agreement with the previous studies. The energy of the spectrum knee is about $\sim 150 \mathrm{keV}$ for typical conditions of the Earth magnetotail. We compare theoretical results with the spacecraft observations of electron double power-law spectra in the magnetotail and demonstrate that the theory is able to describe typical energy of the spectra knee. We also estimate the role of relativistic effects and magnetic field fluctuations on the electron acceleration: the acceleration is more stable for relativistic electrons, while fluctuations of the magnetic field cannot significantly decrease the gained energy for typical magnetospheric conditions.
\end{abstract}

Keywords. Magnetospheric physics (Magnetotail; Storms and substorms) - Space plasma physics (Charged particle motion and acceleration)

\section{Introduction}

Charged particle acceleration in the course of magnetic reconnection is considered now as one of the main mechanisms producing high-energy electrons in the magnetotail current sheet (Hoshino, 2005; Drake et al., 2006; Pritchett, 2006;
Oka et al., 2010) and in solar flares (see Hannah and Fletcher, 2006; Litvinenko, 2006; Anastasiadis et al., 2008; Zharkova et al., 2011, and references therein). Although, electron acceleration in the magnetotail X-line was investigated in several targeted experimental studies (see, e.g., Imada et al., 2007, 2011; Retinò et al., 2008; Asano et al., 2010; Vaivads et al., 2011) and numerical modellings (see, e.g., Drake et al., 2006; Pritchett, 2006), many features of electron energy distributions are still uninvestigated.

The most straightforward method to study the electron acceleration in the vicinity of the X-line is numerical simulation with self-consistent electromagnetic fields, when electron distribution is shaped by calculations of numerous testparticle trajectories (Hoshino, 2005; Drake et al., 2006; Pritchett, 2006; Divin et al., 2010). This method accounts for many features of acceleration, however, it cannot directly separate various mechanisms and estimate their effectiveness. Better physical insight could be given by development of an analytical theory of charge particle dynamics and acceleration in the vicinity of the X-line. Although, such a theory takes into account only few details of the acceleration process and presents results in substantially simplified form, it is capable of providing us with scalings and estimates of typical energies.

The analytical models of charged particle dynamics and acceleration in the X-line geometry can be divided into two groups. The first group consists of the models of the stationary X-line with the external electric field, while the models from the second group consider dynamical development of the $\mathrm{X}$-line with the corresponding growth of induction electric fields (see brief comparison of these two approaches in 
review by Birn et al., 2012). The time scale of such X-line formation in the magnetotail is a few minutes, i.e., it is comparable with the time scale of ion motion (see, e.g., Birn et al., 2001). As a result, even for the dynamical regime of the reconnection, the electron acceleration can be considered as a quasi-stationary process. However, the electric field amplitude should be increased due to input of induction fields.

Since the first papers by Bulanov and Sasorov (1976) and Galeev (1979), charged particle motion in the vicinity of the $\mathrm{X}$-line was recognised as unstable. This instability results in a finite time, which particles spend in the acceleration region. Corresponding energy gain is also limited (see, e.g., Zelenyi et al., 1990a; Burkhart et al., 1990; Moses et al., 1993; Vekstein and Priest, 1995; Litvinenko, 2006). However, as was shown by Hoshino (2005) electrostatic fields transverse to the $\mathrm{X}$-line can create local potential minima for electrons. Trapping of electrons in these potential wells substantially increases the acceleration time. The effect of the trapped electron acceleration was also studied for the magnetic reconnection with guide field configuration by Egedal et al. (2008, see also references therein). It is similar to the well known effect of the charged particle capture by an electrostatic wave (Sagdeev and Shapiro, 1973; Katsouleas and Dawson, 1983) and, thus, was called surfing acceleration (Hoshino, 2005).

Additionally to the direct acceleration in the vicinity of the X-line (where electrons are unmagnetised), other mechanisms of acceleration can be involved. At the right and left flanks of the X-line where the normal component of the magnetic field has a finite value, electrons gain energy due to the Speiser mechanism of acceleration (Speiser, 1967; Shabansky, 1971; Lyons and Speiser, 1982; Ashour-Abdalla et al., 1995). Roughly speaking, both these mechanisms can be considered as an unstable particle acceleration in the neutral plane in the presence of a finite normal component (AshourAbdalla et al., 1993, 1995). Eventually, when electrons penetrate into the region with a strong magnetic field and start drifting away from the X-line, Fermi and betatron mechanisms contribute to the energy gain (Tverskoy, 1969; Lyons, 1984; Zelenyi et al., 1990b). These two mechanisms are responsible for the acceleration of particles with small pitchangles (i.e., with distant mirror points) and with large pitchangles (i.e., trapped inside the current sheet), respectively (see comparison of these mechanisms for protons in the magnetotail in Ashour-Abdalla et al., 1994).

In this paper, we investigate the relation between various mechanisms of the electron acceleration in the vicinity of the $\mathrm{X}$-line. We use analytical models of particle dynamics and numerical calculations of test trajectories. We compare energy distribution of electrons with spacecraft observations in the Earth magnetotail. The major attention is given to observations and modelling of the specific double power-law spectra of high-energy electrons $(>20 \mathrm{keV})$.

\section{Model of electron acceleration in X-line}

In this section, we describe various regimes of the electron acceleration in the vicinity of the X-line. Analytical estimates of gained energy allow us to compare roles of different acceleration mechanisms. As a main result, we obtain the estimate of the energy of the spectrum knee as a function of the system parameters. This value is compared with numerical calculations as well as with spacecraft observations. Each acceleration regime corresponds to the certain spatial region. We consider the magnetic field configuration $\boldsymbol{B}=\sigma B_{0}\left(x / L_{x}\right) \boldsymbol{e}_{\mathrm{z}}+B_{0}\left(z / L_{\mathrm{z}}\right) \boldsymbol{e}_{\mathrm{x}}$, where $B_{0}$ is the magnetic field amplitude, $L_{\mathrm{X}}$ and $L_{\mathrm{z}}$ are spatial scales of magnetic field inhomogeneity along $\mathrm{x}$ - and z-directions, respectively, and the parameter $\sigma$ determines stretching of field lines $(\sigma=$ $\left.\max \left(\boldsymbol{B} \cdot \boldsymbol{e}_{\mathrm{Z}}\right) / \max \left(\boldsymbol{B} \cdot \boldsymbol{e}_{\mathrm{x}}\right) \sim 0.25-0.01\right)$. From simple models of 2-D current sheets, one can estimate $\sigma \approx 2 L_{\mathrm{z}} / L_{\mathrm{x}}$ (see, e.g., Lembège and Pellat , 1982). We also assume that in the spatial domain $\left|x / L_{\mathrm{x}}\right|<1,\left|z / L_{\mathrm{z}}\right|<1$ constant electrostatic field $E_{\mathrm{y}}$ is applied.

Here we consider the single-scale model of the $\mathrm{X}$-line, i.e., gradient of the magnetic field across the neutral plane $z=0$ can be described by one scale, $L_{\mathrm{z}}$. Numerical simulations showed that the $\mathrm{X}$-line region can contain several scales corresponding to different intensities of the current density carried by various particle populations (see, e.g., Hesse et al., 2006; Karimabadi et al., 2007; Divin et al., 2010, and references therein). For example, accelerated electrons can carry strong current, which provides a decrease of the scale $L_{\mathrm{Z}}$ in the vicinity of the X-line (Divin et al., 2012). However, here we assume that population of accelerated electrons is sufficiently small in comparison with the thermal background population. Thus, we restrict our consideration to the simplest case with single $L_{z}$ to derive analytical estimates of electron dynamics and acceleration.

Initial energy of electrons is $\varepsilon_{0}$ and their Larmor radius $\rho_{\mathrm{e}}$ in the top and bottom boundaries is $\rho_{0}=\sqrt{2 \varepsilon_{0} / m_{\mathrm{e}}} / \omega_{0}$, where $\omega_{0}=e B_{0} / m_{\mathrm{e}} c$ and $m_{\mathrm{e}}$ is the electron mass. For simplicity, we consider dynamics of nonrelativistic electrons, while relativistic effects are discussed in Appendix C.

In the vicinity of the $\mathrm{X}$-line, the magnetic field magnitude is small enough to consider electrons as unmagnetised particles. The boundary of this region along the z-direction, $|z|=L_{z}^{\mathrm{nad}}$, can be found from the equation $\rho_{\mathrm{e}}\left(L_{\mathrm{z}}^{\mathrm{nad}}\right)=$ $\rho_{0} /\left(z / L_{\mathrm{z}}\right)=L_{\mathrm{z}}^{\text {nad }}$ (i.e., inside this region electrons are practically not affected by magnetic field $B_{\mathrm{X}}(z)$, see, e.g., Dobrowolny, 1968): $L_{\mathrm{z}}^{\mathrm{nad}}=\sqrt{\rho_{0} L_{\mathrm{z}}}$. This is the boundary of particle meandering oscillations in the inhomogeneous magnetic field $B_{\mathrm{x}}(z) \sim z / L_{\mathrm{z}}$ (see, e.g., Büchner and Zelenyi, 1989). Similarly we estimate the position of the boundary along X-direction: $L_{\mathrm{x}}^{\mathrm{nad}}=\sqrt{\rho_{0} L_{\mathrm{x}} / \sigma}$. In the region $|x|<$ $L_{\mathrm{x}}^{\mathrm{nad}},|z|<L_{\mathrm{z}}^{\mathrm{nad}}$ electrons are non-adiabatic and can gain energy due to the direct acceleration by the electric field $E_{\mathrm{y}}$.

The central region of the $\mathrm{X}$-line is surrounded by stretched magnetic field lines with parabolic form (see schematic 
view in Fig. 1). Particle motion in such a field configuration is determined by $\kappa$ parameter (see Büchner and Zelenyi, 1989). Because $B_{\mathrm{Z}}$ component depends on $x$ we have $\kappa(x)=\left(B_{\mathrm{Z}}(x) / B_{0}\right) \sqrt{L_{\mathrm{Z}} / \rho_{0}}=\left(\sigma x / L_{\mathrm{X}}\right) \sqrt{L_{\mathrm{Z}} / \rho_{0}}$. Thus, we can define the boundary of the region where $\kappa<1$ : $L_{\mathrm{x}}^{\mathrm{qad}}=\left(L_{\mathrm{x}} / \sigma\right) \sqrt{\rho_{0} / L_{\mathrm{z}}}=\sqrt{L_{\mathrm{x}} / \sigma L_{\mathrm{z}}} L_{\mathrm{x}}^{\mathrm{nad}}$. Inside this region electrons are quasi-adiabatic particles which gain energy accordingly to the Speiser mechanism (Speiser, 1965, 1967; Lyons and Speiser, 1982; Ashour-Abdalla et al., 1994, 1995).

In the region $L_{\mathrm{x}}>|x|>L_{\mathrm{x}}^{\mathrm{qad}}$ electrons are magnetised and their motion is adiabatic with conserved magnetic moment and longitudinal invariant. Such particles oscillate along bounce trajectories and move due to the cross-field drift $\sim c E_{\mathrm{y}} / B_{\mathrm{Z}}(x)$ towards the regions with larger magnetic field $B_{\mathrm{Z}}(x)$. As a result, these particles gain energy accordingly to the betatron and Fermi mechanisms (Tverskoy, 1969; Lyons, 1984; Zelenyi et al., 1990b).

In this paper, we also consider the electrostatic field $\boldsymbol{E}=$ $\sigma E_{0}\left(x / L_{\mathrm{x}}\right) \boldsymbol{e}_{\mathrm{x}}-E_{0}\left(z / L_{\mathrm{z}}\right) \boldsymbol{e}_{\mathrm{z}}$. Spatial distribution of components of this field is defined from the numerical simulations (see, e.g., Hoshino, 2005, and references therein). Both components of this field are determined by the scalar potential in the vicinity of the X-line $\varphi(x, z) \sim\left(\varphi_{0} / 2\right)\left(\left(z / L_{\mathrm{Z}}\right)^{2}-\right.$ $\left.\left(x / L_{\mathrm{x}}\right)^{2}\right)$, where $E_{0}=\varphi_{0} / L_{\mathrm{z}}$ and $\sigma \sim L_{\mathrm{z}} / L_{\mathrm{x}}$. This potential is due to the separation of electron and ion motions in the Hall region, where ions can be considered as unmagnetised, while electrons are still adiabatic. Corresponding components of the electric field are $E_{\mathrm{z}} \sim-z, E_{\mathrm{x}} \sim x$ (see, e.g., analytical model by Korovinskiy et al., 2008). The amplitude of the scalar potential $\varphi$ is defined by the pressure balance and can reach $\sim B_{0}^{2} / 4 \pi n_{0} e \sim 1-10 \mathrm{keV}$, where $n_{0}$ is the plasma density in the X-line (Divin et al., 2012).

Equations of motion for nonrelativistic electrons can be written as

$\left\{\begin{array}{l}\dot{v}_{\mathrm{x}}=-\omega_{0} v_{\mathrm{y}}\left(\sigma x / L_{\mathrm{x}}\right)-\omega_{0} w_{\mathrm{D}}\left(\sigma x / L_{\mathrm{x}}\right) \\ \dot{v}_{\mathrm{y}}=-\omega_{0} v_{\mathrm{D}}-\omega_{0}\left(v_{\mathrm{z}}\left(z / L_{\mathrm{z}}\right)-v_{\mathrm{x}}\left(\sigma x / L_{\mathrm{x}}\right)\right) \\ \dot{v}_{\mathrm{z}}=\omega_{0} v_{\mathrm{y}}\left(z / L_{\mathrm{z}}\right)+\omega_{0} w_{\mathrm{D}}\left(z / L_{\mathrm{z}}\right)\end{array}\right.$

where $w_{\mathrm{D}}=c E_{0} / B_{0}$ and $v_{\mathrm{D}}=c E_{\mathrm{y}} / B_{0}$. We introduce the following dimensionless variables and parameters: $\{x, y, z\} / \rho_{0} \rightarrow\{x, y, z\}, \quad\left\{v_{\mathrm{x}}, v_{\mathrm{y}}, v_{\mathrm{z}}\right\} / \omega_{0} \rho_{0} \rightarrow\left\{v_{\mathrm{x}}, v_{\mathrm{y}}, v_{\mathrm{z}}\right\}$, $t \omega_{0} \rightarrow t, \quad\left\{w_{\mathrm{D}}, v_{\mathrm{D}}\right\} / \omega_{0} \rho_{0} \rightarrow\left\{w_{\mathrm{D}}, v_{\mathrm{D}}\right\}, \quad \rho_{0} / L_{\mathrm{z}, \mathrm{x}}=\alpha_{\mathrm{z}, \mathrm{x}}$, $\ell_{\mathrm{z}}=L_{\mathrm{z}}^{\mathrm{nad}} / \rho_{0}=\alpha_{\mathrm{z}}^{-1 / 2}, \quad \ell_{\mathrm{x}}=L_{\mathrm{x}}^{\mathrm{nad}} / \rho_{0}=\left(\sigma \alpha_{\mathrm{x}}\right)^{-1 / 2}$, $\hat{\ell}_{\mathrm{x}}=L_{\mathrm{x}}^{\mathrm{qad}} / \rho_{0}=\alpha_{\mathrm{z}}^{1 / 2}\left(\sigma \alpha_{\mathrm{x}}\right)^{-1}$. Dimensional electron energy is $\varepsilon / \varepsilon_{0} \rightarrow \varepsilon$. In this case one can write

$$
\left\{\begin{array}{l}
\dot{v}_{\mathrm{x}}=-\alpha_{\mathrm{x}} \sigma x\left(v_{\mathrm{y}}+w_{\mathrm{D}}\right) \\
\dot{v}_{\mathrm{y}}=-v_{\mathrm{D}}-\left(\alpha_{\mathrm{z}} v_{\mathrm{z}} z-\alpha_{\mathrm{x}} \sigma v_{\mathrm{x}} x\right) \\
\dot{v}_{\mathrm{z}}=\alpha_{\mathrm{z}} z\left(v_{\mathrm{y}}+w_{\mathrm{D}}\right)
\end{array}\right.
$$

If we assume that the initial current sheet thickness $L_{\mathrm{Z}}$ is about proton Larmor radius $\rho_{0 \mathrm{p}}$, then $\alpha_{\mathrm{z}}^{-1}=\rho_{0 \mathrm{p}} / \rho_{0}=$ $\sqrt{T_{\mathrm{p}} m_{\mathrm{p}} / T_{\mathrm{e}} m_{\mathrm{e}}} \approx 10^{2}$ for typical conditions of the Earth magnetotail where the ratio of proton to electron temperature is $T_{\mathrm{p}} / T_{\mathrm{e}} \sim 3-5$ (Artemyev et al., 2011a). The factor of

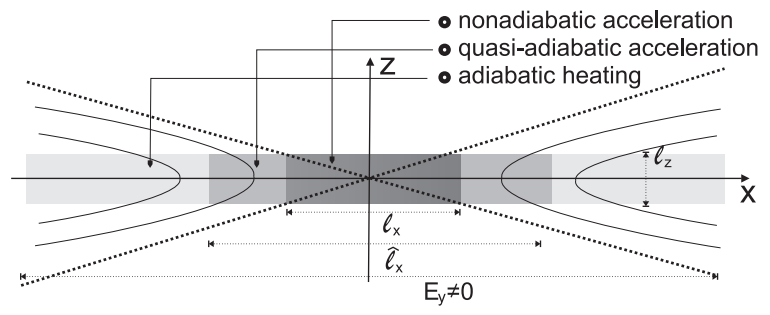

Fig. 1. Scheme of the modelling domain.

$\mathrm{X}$-line stretching gives $L_{\mathrm{X}} / L_{\mathrm{Z}} \sim 10-100$ and correspondingly $\alpha_{\mathrm{x}}^{-1} \sim 10^{3}-10^{4}$. For the typical $\mathrm{X}$-line in the magnetotail we have $E_{\mathrm{y}} \sim 1-10 \mathrm{mV} \mathrm{m}^{-1}$ and $E_{0} / E_{\mathrm{y}} \sim 10^{2}$ (see, e.g., observations and modelling by Wygant et al., 2005; Hoshino, 2005). Thermal velocity of magnetotail electrons is $\sqrt{2 \varepsilon_{0} / m_{\mathrm{e}}} \sim 10^{4} \mathrm{~km} \mathrm{~s}^{-1}$ and the amplitude of the magnetic field is $B_{0} \sim 10 \mathrm{nT}$. As a result, we can estimate the parameter $v_{\mathrm{D}} \sim 10^{-2}-10^{-1}$. Parameters $\ell_{\mathrm{x}}$ and $\hat{\ell}_{\mathrm{x}}$ are $\sim 10^{2}-10^{3}$ and $\sim 10^{3}-10^{4}$, respectively. The schematic view of all spatial scales in the system is shown in Fig. 1.

To estimate typical energies of electron acceleration, one should introduce the initial distribution of cold particles. According to common scheme, particles are coming into the reconnection region due to $\sim E_{\mathrm{y}} / B_{\mathrm{x}}$ drift from the inflow regions located above and below the $\mathrm{X}$-line. Also certain population moving along field lines can approach the $\mathrm{X}$-line from the flanks (Ashour-Abdalla et al., 1995). Here we would like to estimate the maximal possible energy gained by electrons. Thus, we simplify our initial conditions and locate all particles directly to the X-line, while initial particle velocity $\dot{x}(0)$ is about the thermal electron velocity. Such initial conditions restrict our analysis by electrons dropping exactly to the Xline from the bottom and top inflow regions, while nonzero $\dot{x}(0)$ provides realistic estimates of energy gain due to natural limitation of the acceleration time.

\subsection{Acceleration in the non-adiabatic region}

Let us consider electron acceleration in the region $|x|<\ell_{\mathrm{x}}$, $|z|<\ell_{\mathrm{Z}}$. Firstly, we consider the system without electrostatic fields $w_{\mathrm{D}}=0$. One can neglect the terms $\sim z$ and $\sim x$ in the second equation of system (1) and obtain expression $v_{\mathrm{y}} \approx-t v_{\mathrm{D}}$. Substituting this expression into first and second Eqs. (1) we obtain:

$\left\{\begin{array}{l}\ddot{x}=t v_{\mathrm{D}} \alpha_{\mathrm{x}} \sigma x \\ \ddot{z}=-t v_{\mathrm{D}} \alpha_{\mathrm{z}} z\end{array}\right.$

This system describes electron dynamics in the vicinity of the $\mathrm{X}$-line. Solution of this system for the initial condition $x(0)=0, z(0)=0$ is

$$
\begin{aligned}
& x(t)=\text { const } \cdot\left(\operatorname{Bi}\left(t \alpha_{\mathrm{x}}^{1 / 3} \sigma^{1 / 3} v_{\mathrm{D}}^{1 / 3}\right)-\sqrt{3} \mathrm{Ai}\left(t \alpha_{\mathrm{x}}^{1 / 3} \sigma^{1 / 3} v_{\mathrm{D}}^{1 / 3}\right)\right) \\
& z(t)=\mathrm{const} \cdot\left(\operatorname{Bi}\left(-t \alpha_{\mathrm{Z}}^{1 / 3} v_{\mathrm{D}}^{1 / 3}\right)-\sqrt{3} \operatorname{Ai}\left(-t \alpha_{\mathrm{z}}^{1 / 3} v_{\mathrm{D}}^{1 / 3}\right)\right)
\end{aligned}
$$



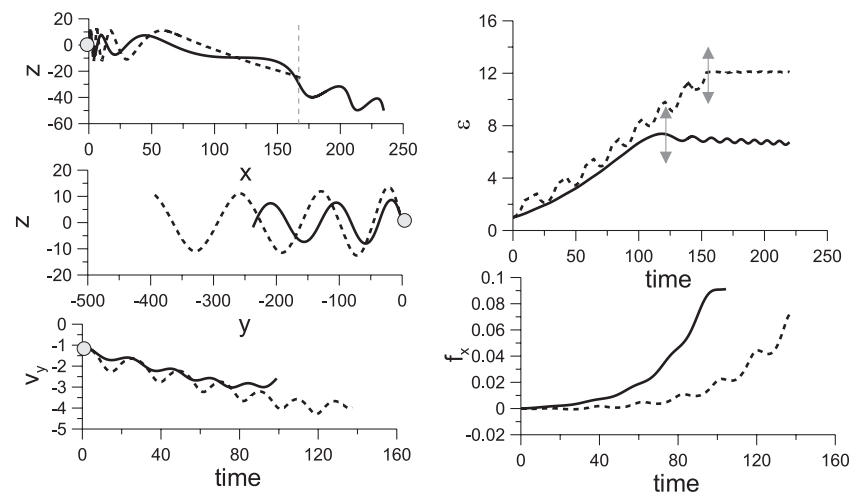

Fig. 2. Two particle trajectories in the system with $w_{\mathrm{D}}=0$ (solid curve) and in the system with $w_{\mathrm{D}}=1.5$ (dotted curve). The gray circles show initial positions of particles. Gray dotted line and grey arrows show moment when particles reach the boundary $|x|=\ell_{\mathrm{X}}$.

where $\mathrm{Bi}$ and $\mathrm{Ai}$ are the Airy wave functions (see, e.g., Divin et al., 2010; Birn et al., 2012, and references therein). $z(t)$ oscillates, while an amplitude of $x(t)$ exponentially grows with time. Therefore, inside this region particles can spend only a finite time interval $T^{\text {nad }}$ defined by the equation $x\left(T^{\text {nad }}\right) \approx \ell_{\mathrm{x}}$. We use expansion $\quad x(t) \approx \dot{x}(0) t\left(\alpha_{\mathrm{x}} \sigma v_{\mathrm{D}}\right)^{1 / 3}+\dot{x}(0) c_{\mathrm{x}} t^{4}\left(\alpha_{\mathrm{x}} \sigma v_{\mathrm{D}}\right)^{4 / 3}+.$. where constant $c_{\mathrm{x}}=\Gamma(2 / 3) 3^{2 / 3} / 12 \pi \approx 0.1$ and $\Gamma$ is the gamma function. Assuming the first term to be small in comparison with the second term, we obtain $T^{\text {nad }}=\left(\ell_{\mathrm{x}} / \dot{x}(0) c_{\mathrm{x}}\right)^{1 / 4}\left(\alpha_{\mathrm{x}} \sigma v_{\mathrm{D}}\right)^{-1 / 3}$. In this case the maximal gain of energy is $\varepsilon_{\text {max }}^{\text {nad }}=\left(T^{\text {nad }} v_{\mathrm{D}}\right)^{2}=$ $\left(\ell_{\mathrm{x}} / \dot{x}(0) c_{\mathrm{x}}\right)^{1 / 2}\left(\alpha_{\mathrm{x}} \sigma\right)^{-2 / 3} v_{\mathrm{D}}^{4 / 3} \approx c_{\mathrm{x}}^{-1 / 2}\left(\alpha_{\mathrm{x}} \sigma\right)^{-11 / 12} v_{\mathrm{D}}^{4 / 3}$, where we assume $\dot{x}(0) \sim 1$. For typical magnetotail parameters, we obtain $T^{\text {nad }} \sim 150-300$ and $\varepsilon_{\max }^{\text {nad }} \sim 10-100$.

In the system with the electrostatic field $w_{\mathrm{D}} \neq 0$, we can rewrite Eqs. (2) as:

$\left\{\begin{array}{l}\ddot{x}=-\alpha_{\mathrm{X}} \sigma\left(w_{\mathrm{D}}-t v_{\mathrm{D}}\right) x \\ \ddot{z}=\alpha_{\mathrm{z}}\left(w_{\mathrm{D}}-t v_{\mathrm{D}}\right) z\end{array}\right.$

Therefore, particles can spent time $\sim T^{\mathrm{nad}}+w_{\mathrm{D}} / v_{\mathrm{D}}$ in the vicinity of the $\mathrm{X}$-line. So, for typical conditions of the reconnection region in the magnetotail we have $w_{\mathrm{D}} / v_{\mathrm{D}} \sim$ $(2 / 3) T^{\text {nad }}-(1 / 2) T^{\text {nad }}$. Thus, the maximum energy gain can be estimated as $\varepsilon_{\max }^{\text {nad }} \sim 20-200$.

We support our estimates by numerical calculations of particle trajectories (description of the numerical scheme can be found in Appendix A). Figure 2 demonstrates two trajectories for the system without electrostatic field and with $w_{\mathrm{D}}=1.5$. We show projections of the particle trajectories onto $(x, z)$ and $(y, z)$ planes and dependencies of $v_{\mathrm{y}}$ on time. One can see, that the presence of a finite $w_{\mathrm{D}}$ results in the increase of energy gain and time, which the particle spends in the vicinity of the $\mathrm{X}$-line. Component of the Lorentz force $f_{\mathrm{x}}=-\alpha_{\mathrm{x}} \sigma x\left(v_{\mathrm{y}}+w_{\mathrm{D}}\right)$ grows slowly with time for the system

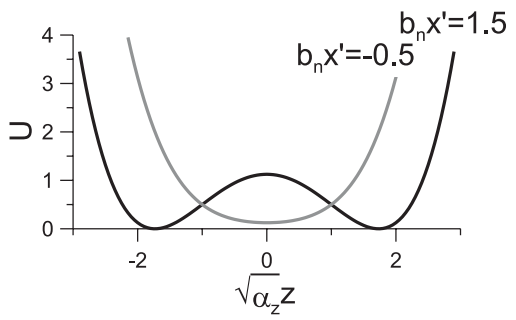

Fig. 3. Profiles of potential energy $U\left(b_{\mathrm{n}} x^{\prime}, z\right)=\frac{1}{2}\left(b_{\mathrm{n}} x^{\prime}-\frac{1}{2} \alpha_{\mathrm{z}} z^{2}\right)^{2}$ for two values of $b_{\mathrm{n}} x^{\prime}$ parameter.

with $w_{\mathrm{D}} \neq 0$ in comparison with the system without electrostatic field.

\subsection{Acceleration in the quasi-adiabatic region}

Here we consider electron dynamics in the region $\hat{\ell}_{\mathrm{x}}>|x|>$ $\ell_{\mathrm{x}}$. In this region electrons move along Speiser trajectories. Without loss of generality, we consider $x>0$, where $B_{Z}(x)>0$. In absence of the electrostatic field system (1) can be written as

$\left\{\begin{array}{l}\dot{v}_{\mathrm{x}}=-b_{\mathrm{n}} v_{\mathrm{y}} \\ \dot{v}_{\mathrm{y}}=-v_{\mathrm{D}}-\left(\alpha_{\mathrm{z}} v_{\mathrm{z}} z-b_{\mathrm{n}} v_{\mathrm{x}}\right) \\ \dot{v}_{\mathrm{z}}=\alpha_{\mathrm{z}} z v_{\mathrm{y}}\end{array}\right.$

where $b_{\mathrm{n}}=\alpha_{\mathrm{x}} \sigma x \approx$ const $\ll 1$, because electron gyroradius in the neutral plane $\sim \rho_{0} / b_{\mathrm{n}}$ is substantially smaller than $L_{\mathrm{x}}$. We introduce new variables $v_{\mathrm{x}}^{\prime}=v_{\mathrm{x}}-v_{\mathrm{D}} / b_{\mathrm{n}}, x^{\prime}=x-$ $v_{\mathrm{D}} t / b_{\mathrm{n}}+\dot{y}_{0}$, where $\dot{y}_{0}$ is an initial value of $v_{\mathrm{y}}$. Then we integrate the second equation of system (3) and substitute it into two other equations:

$\left\{\begin{array}{l}\ddot{x}^{\prime}+b_{\mathrm{n}}^{2} x^{\prime}=\frac{1}{2} \alpha_{\mathrm{z}} b_{\mathrm{n}} z^{2} \\ \ddot{z}=\alpha_{\mathrm{z}} z\left(b_{\mathrm{n}} x^{\prime}-\frac{1}{2} \alpha_{\mathrm{z}} z^{2}\right)\end{array}\right.$

Because $b_{\mathrm{n}} \ll 1$ variables $\left(b_{\mathrm{n}} x^{\prime}, v_{\mathrm{x}}^{\prime}\right)$ are slow in comparison with variables $\left(z, v_{\mathrm{Z}}\right)$. Assuming $x^{\prime}$ to be constant, we integrate the second equation of system (4)

$\frac{1}{2} v_{\mathrm{z}}^{2}=$ const $+\frac{1}{2}\left(b_{\mathrm{n}} x^{\prime}-\frac{1}{2} \alpha_{\mathrm{z}} z^{2}\right)^{2}$

$z$ coordinate oscillates in the potential well $U\left(b_{\mathrm{n}} x^{\prime}, z\right)=$ $\frac{1}{2}\left(b_{\mathrm{n}} x^{\prime}-\frac{1}{2} \alpha_{\mathrm{z}} z^{2}\right)^{2}$. Profiles of $U\left(b_{\mathrm{n}} x^{\prime}, z\right)$ for various $x^{\prime}$ are shown in Fig. 3. Electrons can oscillate in one of two possible potential wells, when average $z$ is not equal to zero (these oscillations correspond to electron motion far from the neutral plane), or can oscillate inside the single potential well (i.e., across the neutral plane) with average $z=0$ (more detailed description of particle dynamics in the system under consideration can be found in Sonnerup, 1971; Büchner and Zelenyi, 1986, 1989; Chen, 1992).

In the vicinity of the neutral plane electrons oscillate across $z=0$. After averaging over fast variables $\left(z, v_{z}\right)$ for the first equation of system (4) we have $x^{\prime} \sim$ const $\cdot \sin \left(b_{\mathrm{n}} t+\right.$ 
const), i.e., $x^{\prime}$ changes periodically. Therefore, the capture into the region of oscillations inside the single potential well and escape from this regime occur with the same value of $x^{\prime}$ and, as a result, with opposite values of $v_{\mathrm{x}}^{\prime}: v_{\mathrm{x}, \text { escape }}^{\prime}=$ $-v_{\mathrm{x}, \text { capture }}^{\prime}$. Because $v_{\mathrm{x}, \text { capture }}^{\prime}=v_{\mathrm{x}, \text { capture }}-v_{\mathrm{D}} / b_{\mathrm{n}}$ one can write $v_{\mathrm{x}}$, escape $=-v_{\mathrm{x}, \text { capture }}+2 v_{\mathrm{D}} / b_{\mathrm{n}}$, i.e., particle interaction with the current sheet results in the energy gain

$$
\begin{aligned}
& \Delta \varepsilon=\frac{1}{2}\left(\sqrt{2 \varepsilon_{\text {capture }}}+2 v_{\mathrm{D}} / b_{\mathrm{n}}\right)^{2}-\varepsilon_{\text {capture }} \\
& =2\left(v_{\mathrm{D}} / b_{\mathrm{n}}\right) \sqrt{2 \varepsilon_{\text {capture }}}+2\left(v_{\mathrm{D}} / b_{\mathrm{n}}\right)^{2}
\end{aligned}
$$

where $v_{\mathrm{x}, \text { capture }} \approx-\sqrt{2 \varepsilon_{\text {capture }}}$. Such energy gain on Speiser orbit could be understood by the simple analogy with the interaction of an elastic body with a moving wall (Speiser, 1967; Shabansky, 1971; Cowley and Shull Jr., 1983).

Here we also can notice, that for the system with electrostatic field $w_{\mathrm{D}} \neq 0$ one can easily obtain the same energy gain using the shift $v_{\mathrm{y}} \rightarrow v_{\mathrm{y}}+w_{\mathrm{D}}$, i.e., the drift $E_{\mathrm{z}} / B_{\mathrm{x}}$ does not change the energy gain. Therefore, the final energy varies with the $\mathrm{x}$-coordinate of the region, where electrons interact with the current sheet: $\varepsilon^{\mathrm{qad}} \in \frac{1}{2}\left[\left(\sqrt{2 \varepsilon_{\text {capture }}}+\right.\right.$ $\left.\left.2 v_{\mathrm{D}} / \alpha_{\mathrm{Z}}^{1 / 2}\right)^{2},\left(\sqrt{2 \varepsilon_{\text {capture }}}+2 v_{\mathrm{D}} /\left(\sigma \alpha_{\mathrm{x}}\right)^{1 / 2}\right)^{2}\right]$ (due to $b_{\mathrm{n}}=$ $\alpha_{\mathrm{x}} \sigma x$ and $\left.x \in\left[\hat{\ell}_{\mathrm{x}}, \ell_{\mathrm{x}}\right]\right)$. Here $\varepsilon_{\text {capture }}=\varepsilon^{\text {nad }}$ for electrons coming from the region of the direct acceleration and $\varepsilon_{\text {capture }}=\varepsilon_{0}$ for other particles.

The numerical example of the typical Speiser trajectory is presented in Fig. 4. We show particle trajectory in 3-D space and projections onto $(x, z)$ and $(y, z)$ planes. Particle energy is shown in a third panel. One can easily distinguish fragment of trajectory looking like a half-rotation in the neutral plane. Corresponding energy gain is shown in bottom panel.

Roughly speaking, the Speiser meichanism of the particle acceleration in the vicinity of X-line gives maximum energy value similar to estimates for the direct acceleration in the nonadiabatic region for the exponential estimate of $T^{\text {nad }} \sim$ $\left(\sigma v_{\mathrm{D}} \alpha_{\mathrm{X}}\right)^{-1 / 3}$. Speiser energy is $\hat{\varepsilon} \sim\left(v_{\mathrm{D}} / b_{\mathrm{n}}\right)^{2}$, where $b_{\mathrm{n}} \sim$ $\alpha_{\mathrm{x}} \sigma \hat{x}$ and $\hat{x}$ corresponds to the boundary of the nonadiabatic region for initially cold particles, i.e., $\hat{x} \sim \hat{\varepsilon}^{1 / 4} \alpha_{\mathrm{z}}^{1 / 2} / \alpha_{\mathrm{x}}$. Electrons can not rotate in the neutral plane closer to the $\mathrm{X}$-line due to the finite amplitude of oscillations around $B_{\mathrm{Z}} \sim 0$. Then we obtain $\hat{\varepsilon} \sim\left(v_{\mathrm{D}}\left(\sigma \alpha_{\mathrm{x}} \hat{\varepsilon}^{1 / 2}\right)^{-1 / 2}\right)^{2}$ and $\hat{\varepsilon} \sim$ $v_{\mathrm{D}}^{4 / 3} /\left(\sigma \alpha_{\mathrm{X}}\right)^{2 / 3} \sim\left(v_{\mathrm{D}} T^{\mathrm{nad}}\right)^{2}$. These estimates show that the Speiser acceleration of cold particles coming to the X-line from the left and right flanks is limited due to the instability of acceleration in the vicinity of the X-line. Moreover, the same estimates for the nonadiabatic region and the Speiser acceleration with $\hat{x} \sim \varepsilon^{1 / 4}$ show that we choose correct approximation of $x(t)$ function. Strictly speaking, we should take into account the increase of electron energy to estimate the position of the $\ell_{\mathrm{x}}$ boundary. However, $x(t)$ has the exponential asymptote for large $t$, and $T^{\text {nad }}$ slightly depends on $\ell_{\mathrm{x}}\left(T^{\text {nad }} \sim \ln \ell_{\mathrm{x}}\right.$, see Bulanov and Sasorov, 1976). Above to estimate $\varepsilon^{\text {nad }}$ we neglect dependence of $\ell_{\mathrm{x}}$ on $\varepsilon$. Since we obtain $\varepsilon^{\text {nad }} \approx \hat{\varepsilon}$, our approximations are correct.
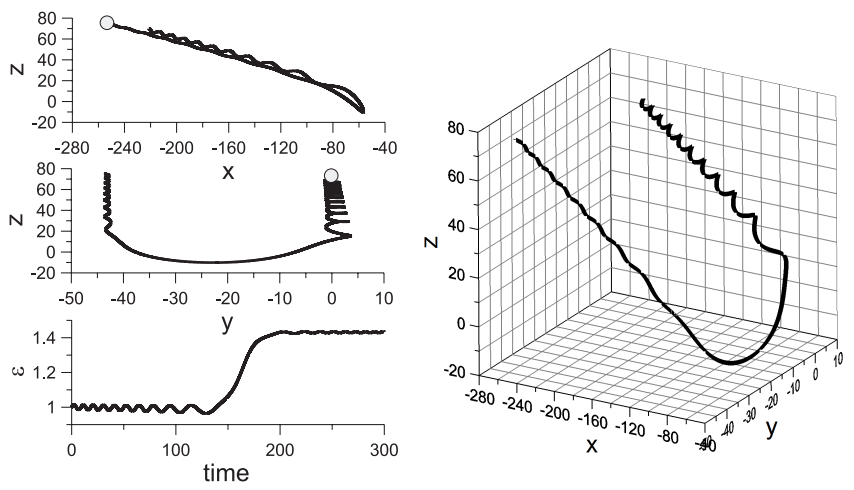

Fig. 4. Trajectory of Speiser particle in the region of quasi-adiabatic motion $\hat{\ell}_{\mathrm{X}}>|x|>\ell_{\mathrm{X}}$. Also energy evolution is shown.

\subsection{Acceleration in the adiabatic region}

When electrons leave the region of quasi-adiabatic motion, they pass through the stochastic region with $\kappa \sim 1$. In this region scattering results in isotropisation of electrons. Then electrons get to the region of the adiabatic motion $(\kappa>1)$ with energy $\varepsilon^{\text {qad }}$. Due to the presence of $E_{\mathrm{y}}$ electrons drift towards the region with spatially growing magnetic field $B_{\mathrm{Z}}(x)$ and gain energy accordingly to the betatron mechanism. As a result, their final energy is

$\varepsilon^{\mathrm{ad}}=\varepsilon^{\mathrm{qad}}\left(1 / \alpha_{\mathrm{x}} \hat{\ell}_{\mathrm{x}}\right)=\left(\sigma / \alpha_{\mathrm{z}}^{1 / 2}\right) \varepsilon^{\mathrm{qad}}$

where $\alpha_{x} \sigma \hat{\ell}_{\mathrm{x}}$ is the magnetic field value at the boundary $x=\hat{\ell}_{\mathrm{x}}$, while $\sigma$ is the magnetic field value at the boundary $|x|=\alpha_{\mathrm{x}}^{-1}$. Therefore, due to the betatron acceleration, electrons can increase their energy by one order of magnitude (for $\sigma=10^{-2}$ ). Here we assume that relatively strong electric field $E_{\mathrm{y}}$ exists until $\left|x / L_{\mathrm{x}}\right|<1$ and all electrons have relatively large pitch-angles to drift in the vicinity of the neutral plane. For the realistic magnetotail condition the region of $E_{\mathrm{y}}$ localisation can be smaller. Moreover, electrons with small pitch-angles gain energy as $\sim B_{\mathrm{z}}^{\lambda}$, where $2 / 5<\lambda<1$ (see, e.g., Tverskoy, 1969; Lyons, 1984; Zelenyi et al., 1990b). As a result, a factor of energy increase $\varepsilon^{\mathrm{ad}} / \varepsilon^{\mathrm{qad}}$ can be smaller than $\sigma / \alpha_{\mathrm{z}}^{1 / 2}$ by a factor of two-three.

Two adiabatic particle trajectories are presented in Fig. 5. We calculate trajectories with the different initial pitchangles and, as a result, with different distances from the neutral plane to mirror points. One can see, that the second trajectory has more distant mirror points and energy gain with time for this particle is lower.

\subsection{Formation of energy distribution}

We obtain two typical values of energy corresponding to the various mechanisms of acceleration:

$$
\begin{aligned}
& \varepsilon_{1} \approx\left(\sqrt{\varepsilon^{\mathrm{nad}}}+\sqrt{2} v_{\mathrm{D}} /\left(\sigma \alpha_{\mathrm{x}}\right)^{1 / 2}\right)^{2} \approx 30-300 \\
& \varepsilon_{2} \approx\left(\sigma / \alpha_{\mathrm{Z}}^{1 / 2}\right) \varepsilon_{1} \approx 50-500
\end{aligned}
$$



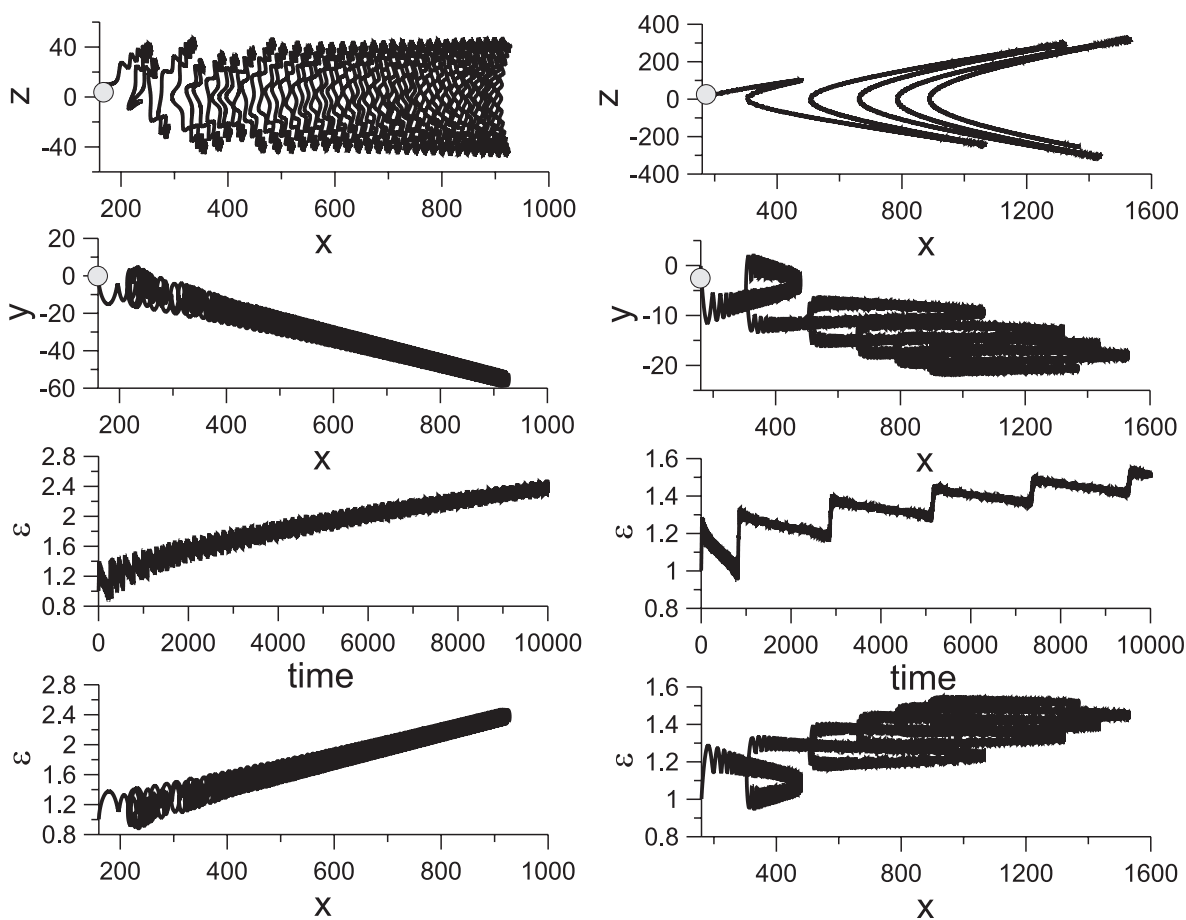

Fig. 5. Two trajectories of adiabatic particles with different initial pitch-angles. Left panels corresponds to large pitch-angle, while right panels show results for small pitch-angle. Also evolutions of the particle energies, $\varepsilon$, with time and as a function of $\mathrm{x}$-coordinate are presented.

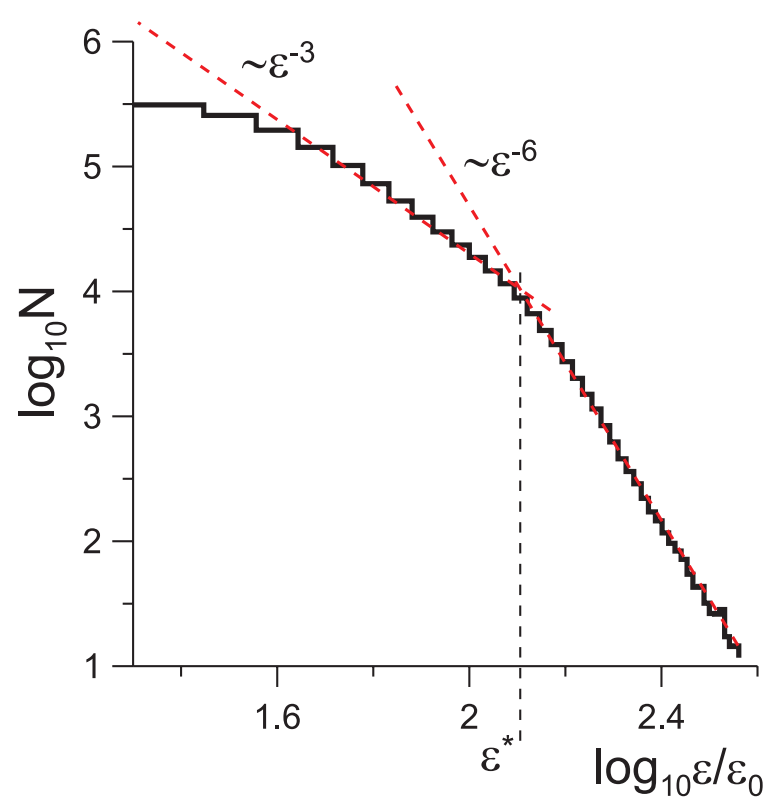

Fig. 6. Energy distribution of particles in the system with $v_{\mathrm{D}}=$ $0.025, w_{\mathrm{D}}=0.5$.

Here $\varepsilon_{1}$ is the energy of electrons after acceleration in the Xline due to capture by the electrostatic field, $\varepsilon_{2}$ corresponds to the additional betatron acceleration. All energies are normalised on the initial value $\varepsilon_{0} \sim 1 \mathrm{keV}$ (actually, direct ac- celeration in the X-line does not depend on the initial particle energy and the normalisation on $\varepsilon_{0}$ comes from the normalisation of spatial parameters on the typical electron Larmor radius). Therefore, for energy distribution we suppose to obtain two fragments $\varepsilon<\varepsilon_{1}$ and $\varepsilon_{1}<\varepsilon<\varepsilon_{2}$ (such double structure of energy distribution has been shown before by Hoshino, 2005). The first fragment corresponds to the electrons accelerated inside the non-adiabatic and quasiadiabatic regions. The second fragment includes particles additionally gain energy due to the adiabatic mechanism. Adiabatic heating is effective only for electrons leaving the quasiadiabatic region with large pitch-angles. Due to geometry of magnetic field lines majority of electrons has small pitchangles (i.e., these accelerated particles move approximately along field lines) and does not contribute to the second fragment of the energy distribution. Therefore, population of particles additionally accelerated by the adiabatic mechanism would be smaller, than population initially accelerated in the $\mathrm{X}$-line. Then the second fragment of energy spectra for $\varepsilon>\varepsilon_{1}$ should be steeper than the fragment $\varepsilon<\varepsilon_{1}$.

We numerically integrate $1.5 \times 10^{6}$ trajectories (see details in Appendix A) and collect energy distribution at the boundary $|x|=\alpha_{\mathrm{x}}^{-1}$ (Fig. 6). One can see the typical double powerlaw structure of the distribution $\sim \varepsilon^{\mathrm{r}}$ with two different $\mathrm{r}$ values. The energy of the knee is $\varepsilon^{*} \sim 140$. The slopes of spectrum are $\sim-3$ and $\sim-6$. If we transform $N(\varepsilon)$ to flux dependence on $\varepsilon$, then corresponding slopes are $r \sim-3.5$ and $r \sim-6.5$. Because $\varepsilon^{*} \sim \varepsilon_{1}$ (for used parameters $\varepsilon_{1} \approx$ 
Table 1. List of events. For the event marked by ${ }^{@}$ Geotail observations show $\left|V_{\mathrm{X}}\right| \sim 300 \mathrm{~km} \mathrm{~s}^{-1}$.

\begin{tabular}{ccccccc}
\hline$N$ & date & $\begin{array}{c}\left|V_{\mathrm{X}}\right| \\
\mathrm{km} \mathrm{s}^{-1}\end{array}$ & $\begin{array}{c}\varepsilon^{*} \\
\mathrm{keV}\end{array}$ & $r_{1}$ & $r_{2}$ & $\begin{array}{c}(X, Y, Z)_{\text {inb }} \\
R_{\mathrm{E}}\end{array}$ \\
\hline 1 & 28 Nov 1995, 10:00-12:00 & $100^{@}$ & 80 & -2 & -4 & $(-12,-2.0,-2.5)$ \\
2 & 15 Dec 1995, 10:02-04:00 & no data & 50 & -2 & -4 & $(-24,9.5,-2.0)$ \\
3 & 23 Dec 1996, 00:30-01:00 & 200 & 90 & -2.5 & -5 & $(-18,10,-4.0)$ \\
4 & 26 Oct 1997, 00:20-00:40 & 400 & 100 & -2 & -8 & $(-24,-13,-2.7)$ \\
5 & 10 Nov 1997, 00:00-00:20 & 300 & 150 & -3 & -6 & $(-25,-7.0,-2.6)$ \\
6 & 21 Oct 1998, 07:40-08:20 & 200 & 150 & -3 & -6 & $(-21,-17,0.5)$ \\
7 & 1 Dec 1998, 15:00-15:40 & 800 & 90 & -3 & -6 & $(-21,-17,0.5)$ \\
8 & 16 Dec 1998, 14:30-16:30 & 800 & 250 & -3 & -6 & $(-24,10,-4.0)$ \\
\hline
\end{tabular}

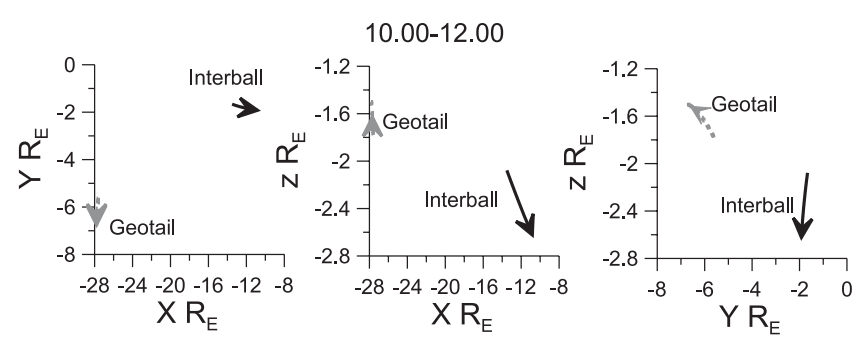

Fig. 7. Positions of Interball-tail and Geotail spacecraft on 28 November 1995 from 10:00 to 12:00.

140, where $\alpha_{\mathrm{z}}=10^{-2}, \alpha_{\mathrm{x}}=10^{-3}, v_{\mathrm{D}}=0.025$, and $w_{\mathrm{D}}=$ $0.5)$, we can conclude that our simplified analytical model describes the main features of the electron acceleration.

\section{Spacecraft observations}

In this paper, we compare our analytical estimates of the electron acceleration with spacecraft observations in the Earth's magnetotail. We find three events of relatively long (duration more than $10 \mathrm{~min}$ ) observations of double power-law spectra by Interball-tail spacecraft in the vicinity of the current sheet neutral plane. DOK2 experiment (Lutsenko et al., 1998) onboard this spacecraft collects energetic electron (and ion) energy distributions with the uniquely high resolution ( $\sim 5-$ $7 \mathrm{keV}$ ) and can distinguish the fine structure of high energy tails (see, e.g., Lutsenko and Kudela, 1999; Artemyev et al., 2012). We use magnetic field from ASPI/MFI-M experiment (Klimov et al., 1997), moments of low-energy ions from CORALL experiment (Yermolaev et al., 1997) and electron density from ELECTRON experiment (Sauvaud et al., 1997). During each event Geotail spacecraft was located in the magnetotail and we present corresponding data of simultaneous observations. We use magnetic field from FGM experiment (Kokubun et al., 1994), moments of low energy ions from LEP experiment (Mukai et al., 1994) and flux of high energy electrons from EPIC experiment (Williams et al., 1994). We also plot magnetic field and plasma velocity observed in solar wind (OMNI data are obtained from CDAWEB site http://cdaweb.gsfc.nasa.gov/). Dates of three events with positions of Interball-tail and Geotail are presented in Table 1. All data are presented in the GSM coordinate system.

Additionally to three long events, we select five events with duration around 1-3 min, when DOK2 also detected electron double power-law spectra (see Table 1, \#4-8). Unfortunately, we did not succeed in finding the simultaneous observations made by Geotail for these five events. Thus, in this paper, we present only eight events of observations of double-power law energy distributions. We restrict our analysis by these events due to their relatively long duration (even short events contain few spectra with the same form). Total statistics of Interball-tail for five years of observations (form 1995 to 1999) includes more examples of double-power law energy distributions detected in the magnetotail. However, major observed events consist of only single double-power law spectrum. Here we discuss only average energy distribution, when the reliability of data is better.

\subsection{Event 28 November 1995, 10:00-12:00}

On 28 November 1995 from 10:00 to 12:00 Interball-tail and Geotail were in the vicinity of the neutral plane of the Earth magnetotail current sheet (Fig. 7). Both spacecraft were at the dawn flank near midnight $\left(Y_{\text {geo }} \approx-6 R_{\mathrm{E}}\right.$ and $Y_{\mathrm{inb}} \approx$ $\left.-2 R_{\mathrm{E}}\right)$. Geotail was in the deep tail with $X_{\mathrm{geo}} \approx-28 R_{\mathrm{E}}$ and Interball-tail was in the near-Earth tail with $X_{\text {inb }} \approx-12 R_{\mathrm{E}}$.

During selected time interval both spacecraft observed two increases of plasma bulk velocity which can be associated with a local magnetic reconnection (see Fig. 8, left column). First event was detected by Geotail as successive observations of earthward (at 10:00-10:15) and tailward (at 10:15-10:40) flows with amplitude around $\sim 400 \mathrm{~km} \mathrm{~s}^{-1}$. During this event Interball-tail was located at some distance from the neutral plane $\left(B_{\mathrm{x}} \sim 20 \mathrm{nT}\right)$ and detected only small variations of ion bulk velocity $<100 \mathrm{~km} \mathrm{~s}^{-1}$. Then around 11:30 Interball-tail observed earthward flow $\sim 300 \mathrm{~km} \mathrm{~s}^{-1}$ and Geotail observed tailward flow $\sim 400 \mathrm{~km} \mathrm{~s}^{-1}$, while both spacecraft approached the neutral plane (this event was indentified as the reconnection pulse and described in details by Petrukovich et al., 1998). During the second event DOK2 
1995.11.28 10.00-12.00
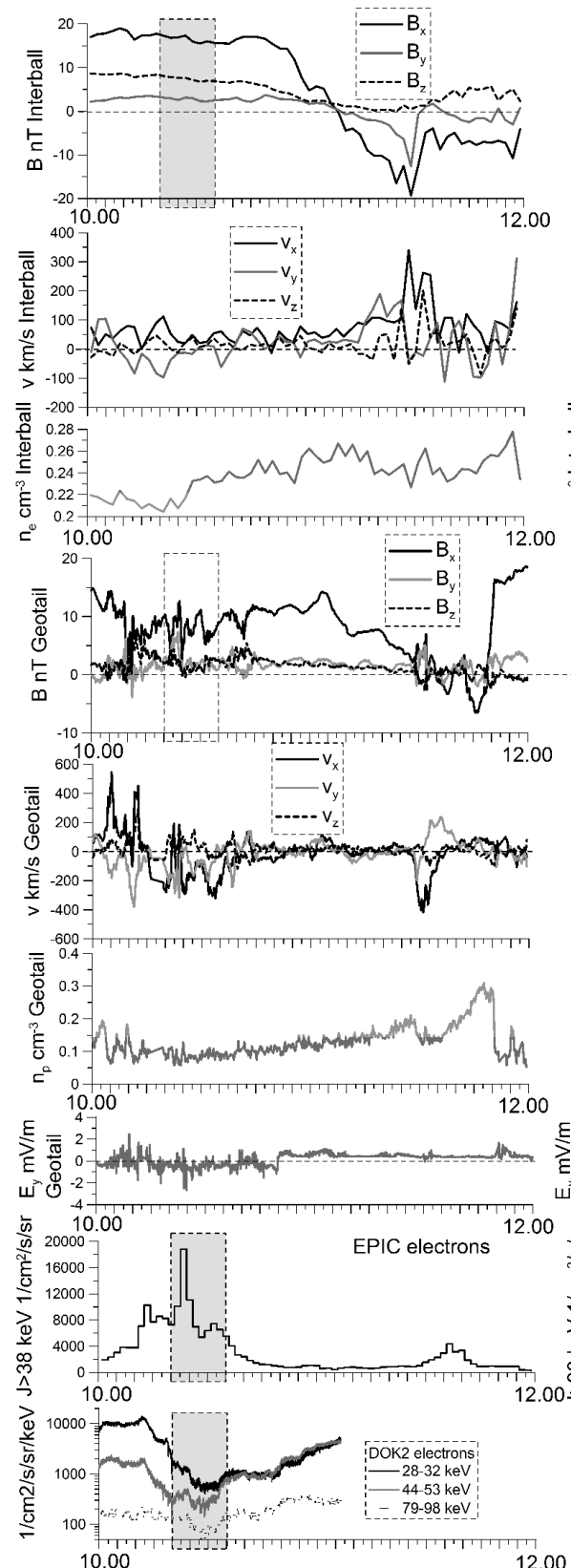
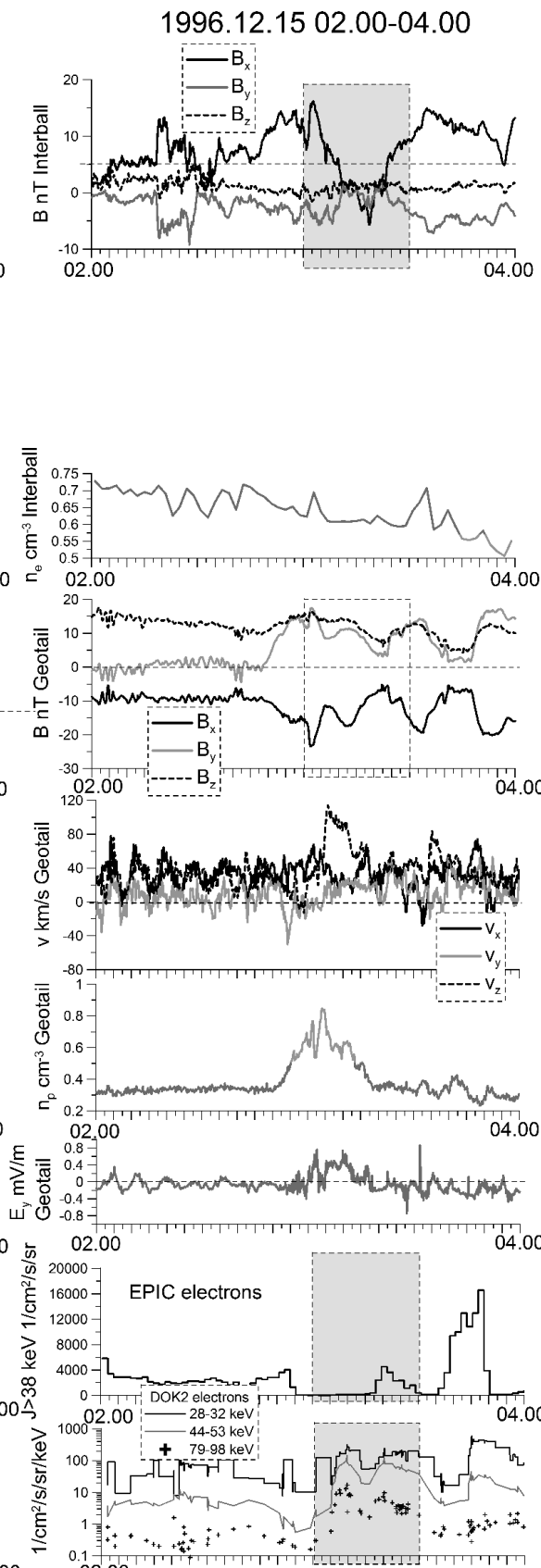

1998.12.16 14.30-16.30
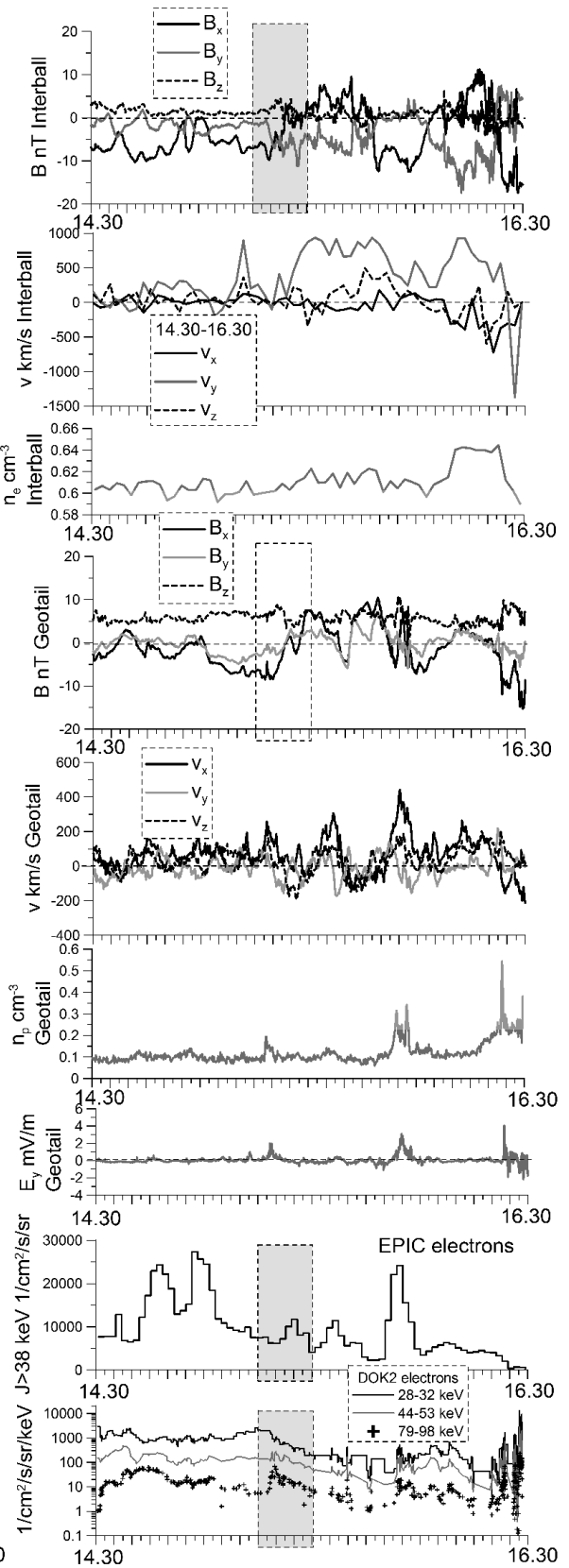

Fig. 8. Magnetic field, ion moments and electron density from Interball-tail and Geotail spacecraft. Fluxes of high-energy electrons from EPIC and DOK2. Periods of observations of double power-law spectra are shown by the colour grey.

instrument onboard Interball-tail did not operate. However, in the first event Interball-tail observed the increase of flux of high-energy electrons (this time period is shown by the colour grey in Fig. 8). Simultaneously EPIC device onboard Geotail detected the increase of flux of high-energy electrons (Fig. 8). Unfortunately, for this period we do not have any data of solar wind conditions.
Fluxes of high-energy electrons collected by DOK2 for three energy ranges (last panel in Fig. 8) show that event is characterised by the flux increase around $50 \mathrm{keV}$ with weaker flux variation for smaller energies. The spectrum of highenergy electrons observed by Interball-tail is presented in Fig. 9a. One can see the double power-law profile with the energy of the knee around $\sim 70-80 \mathrm{keV}$. Such structure of the energy distribution was stably observed during fifteen 

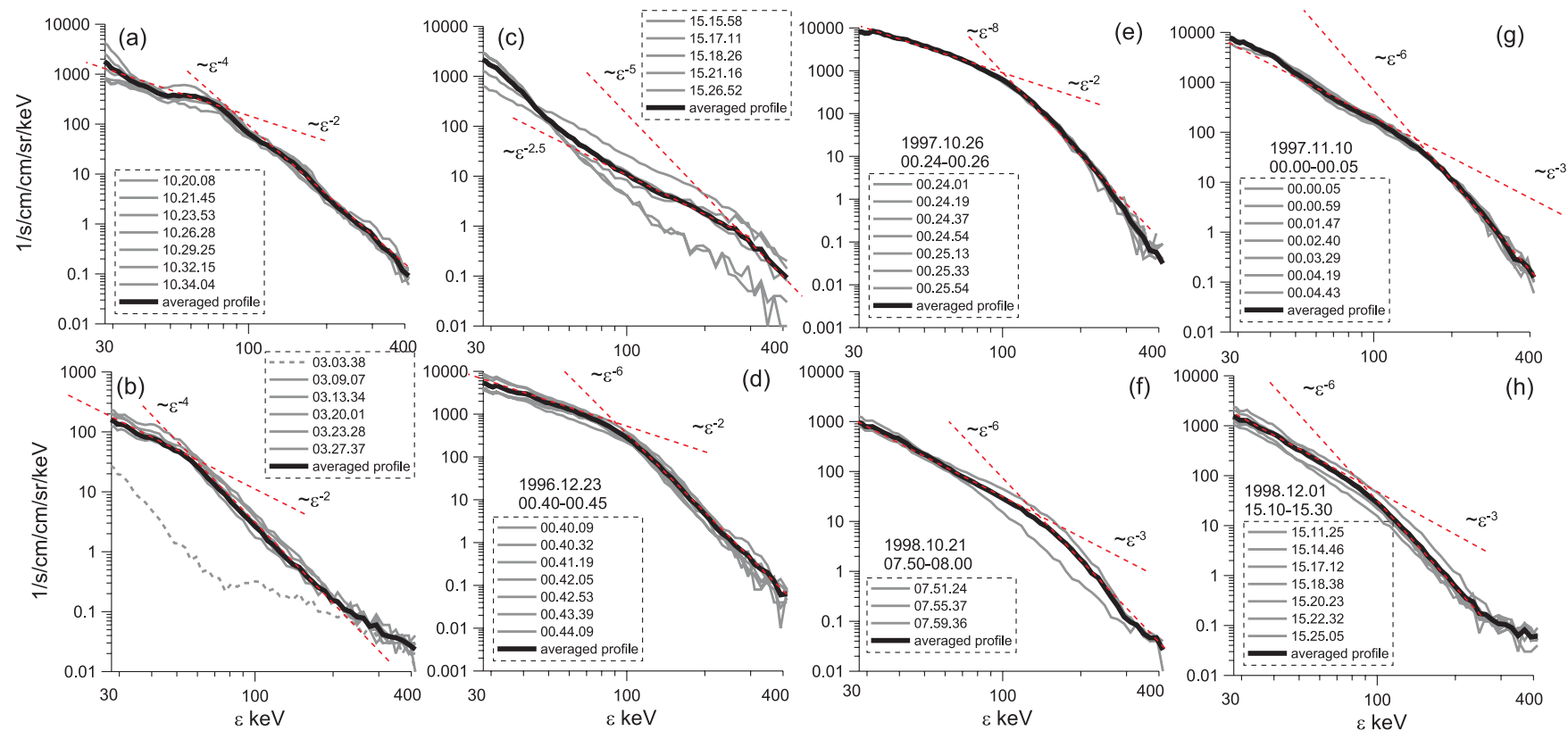

Fig. 9. Spectra of high-energy electrons observed by Interball-tail for three long events 28 November 1995, 10:00-12:00 (a), 15 December 1996, 02:00-04:00 (b), 16 December 1998, 14:30-16:30 (c), and for five short events (dates are presented inside panels).

minutes, while Geotail observed tailward plasma flow. One can also notice the small maximum of flux around $\sim 70 \mathrm{keV}$ pointing out to the multicomponent high-energy electron population coming with fast plasma flow.

Using a technique described by Imada et al. (2011) one can estimate current sheet thickness from vertical pressure balance and particle currents collected by LEP during period 10:00-10:15 and around 11:30. For the first period we obtain current sheet thickness around $10^{4} \mathrm{~km}$ (more than $10 \rho_{0 \mathrm{p}}$ ) and electric field estimation $\boldsymbol{v} \times \boldsymbol{B}$ gives $2 \mathrm{mV} \mathrm{m}^{-1}$. These values are by one order of magnitude larger (for current sheet thickness) and smaller (for electric field) in comparison with the direct observations in the vicinity of the active X-line (see, e.g., Wygant et al., 2005; Retinò et al., 2008; Vaivads et al., 2011). Therefore, here we likely observe particle flow coming from a distant reconnection region rather than local acceleration process.

\subsection{Event 15 December 199, 02:00-04:00}

On 15 December 1996 from 02:00 to 04:00 Interball-tail and Geotail were in the vicinity of the neutral plane of the Earth magnetotail current sheet (Fig. 10). Both spacecraft were at the dusk flank $\left(Y_{\text {geo }} \approx 12 R_{\mathrm{E}}\right.$ and $\left.Y_{\text {inb }} \approx 9 R_{\mathrm{E}}\right)$. Interball-tail was in $X_{\text {inb }} \approx-24 R_{\mathrm{E}}$ and Geotail was in the near-Earth tail with $X_{\text {geo }} \approx-8 R_{\mathrm{E}}$. However, $Y_{\text {geo }}$ coordinate is larger than $X_{\text {geo }}$ and Geotail was located at a deep flank. Before 03:30 conditions of solar wind correspond to the negative $B_{\mathrm{Z}}$ component with two successive reversals (Fig. 10).

From 03:00 to 03:30 Interball-tail crossed the neutral plane (data are shown in Fig. 8, middle column). Unfortunately,

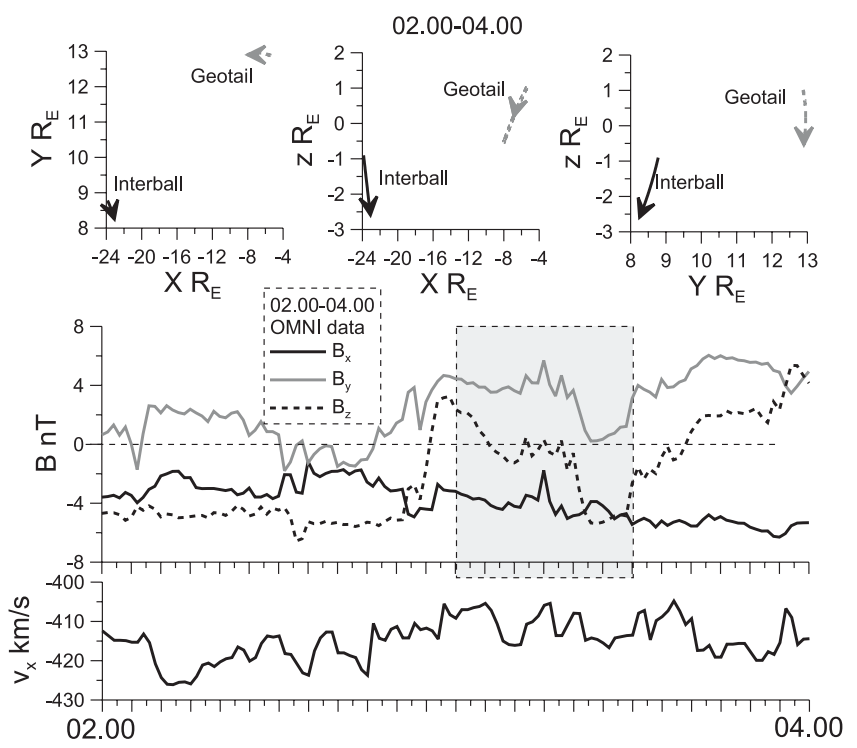

Fig. 10. Positions of Interball and Geotail spacecraft on 15 December 1996 from 02:00 to 04:00. OMNI data: magnetic field and solar wind velocity.

CORALL device was not operating during this time interval and we could not recover the activity level. During the crossing of the neutral plane DOK2 onboard Interball-tail observed the increase of flux of high-energy electrons (this time period is shown by the colour grey in Fig. 8). During time period 03:00-03:30 Geotail observed an increase of plasma density and bulk velocity along the Z-direction. This flow 

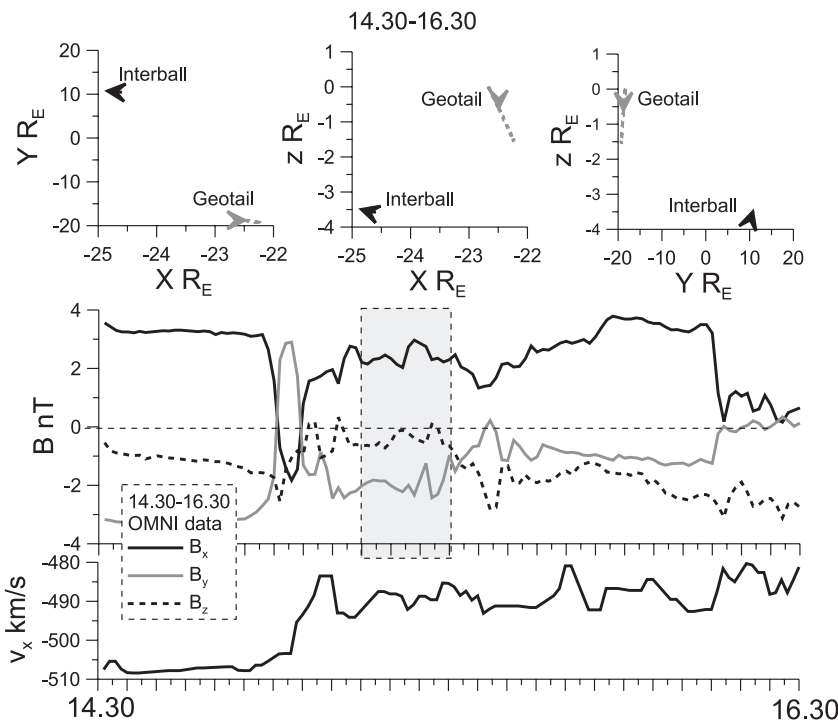

Fig. 11. Positions of Interball-tail and Geotail spacecraft on 15 December 1996 from 02:00 to 04:00. OMNI data: magnetic field and solar wind velocity.

could have no relation to observations of high-energy electrons by Interball-tail, because Geotail was located at the distance of $4 R_{\mathrm{E}}$ from Interball-tail dawnward. EPIC instrument demonstrated only a weak increase of electron flux for this event (Fig. 8). Therefore, this event has substantially poorer in information in comparison with the previous one.

As in the previous event, increase of the flux of highenergy electrons detected by DOK2 was at the energy $\sim 50 \mathrm{keV}$ without similar variations of smaller energy fluxes. For this event, the observed spectrum of high-energy electrons in Interball-tail is presented in Fig. 9b. One can see the double power-law profiles with the energy of the knee $\sim 50 \mathrm{keV}$. Duration of observations of this double power-law spectrum is about twenty minutes.

\subsection{Event 16 December 1998, 14:30-16:30}

On 16 December 1998 from 14:30 to 16:30, Interball-tail and Geotail were in the vicinity of the neutral plane at different flanks (Fig. 11): Interball-tail was in the dusk flank $\left(Y_{\mathrm{inb}} \approx 10 R_{\mathrm{E}}\right)$, while Geotail was in the dawn flank $\left(Y_{\text {geo }} \approx-20 R_{\mathrm{E}}\right)$. The separation of spacecraft along EarthSun direction is only $\sim 2 R_{\mathrm{E}}\left(X_{\mathrm{geo}} \approx-22.5 R_{\mathrm{E}}\right.$ and $X_{\text {inb }} \approx$ $-24.5 R_{\mathrm{E}}$ ). Due to large difference between $Y_{\mathrm{inb}}$ and $Y_{\mathrm{geo}}$ coordinates conjugate observations of two spacecraft were impossible, but we still can use Geotail data to estimate the general level of activity. During this period there was negative $B_{Z}$ in the solar wind (Fig. 11).

Starting from 15:00 and up to 16:30 Interball-tail and Geotail observed sporadic increases of plasma flows with amplitudes around $400-500 \mathrm{~km} \mathrm{~s}^{-1}$ (data are in Fig. 8, right column). During the neutral plane crossing Interball-tail ob- served the increase of flux of high-energy electrons (this time period is shown by the colour grey in Fig. 8). Component $B_{\mathrm{Z}}$ changed its sign and $B_{\mathrm{y}}$ was relatively large. CORALL detected fast ion flows (mainly along dawn-dusk direction). Geotail was far from Interbal-tail and could not detect the same event. However, during time period under consideration, Geotail observed fast earthward/tailward flows. EPIC demonstrated periodical increases of electron flux for this time interval.

Increase of flux of high-energy electrons at Interball-tail position corresponds now to energy around $\sim 100 \mathrm{keV}$ (compare with observations shown for two previous events in Fig. 8). The spectrum of high-energy electrons observed by DOK2 is presented in Fig. 9c. One can see the double powerlaw profiles with the energy of the knee $\sim 280 \mathrm{keV}$.

\subsection{Short time events}

During periods inside the plasma sheet Interball-tail always observed small-scale (duration less than $5 \mathrm{~min}$ ) fast plasma flows. Around these observations DOK2 often detected increases of high-energy electron fluxes. While above we present three events when the observations of high-energy electrons can be associated with plasma flows due to long duration of events (more than $10 \mathrm{~min}$ ), such comparison between proton flows and electron fluxes is not straightforwardly appropriate for short time events, when variations of electron fluxes take less than five minutes. In this section, we show such observations of electron flux increases without strong connection to plasma flows. However, all events were observed in the vicinity of the neutral plane during active period, i.e., within several minutes Interball-tail observed proton flows with amplitude $\sim 300 \mathrm{~km} \mathrm{~s}^{-1}$. Due to shortness of events there is no possibility to find any correlation between Interball-tail and Geotail observations.

High-energy electron spectra are presented in Fig. 9 (panels d-h). One can see typical double power-law spectra with the energy of the knee around $\sim 100 \mathrm{keV}$ for all events. Indexes of energy distribution $\sim \varepsilon^{r}$ is about $r \sim-6$ for the second slope and $r \sim-3$ for the first slope. All profiles are obtained by averaging in time several consecutively collected spectra. All slopes and knee energies are summarised in Table 1 .

\section{Discussion}

Double power-law spectra observed in the magnetotail (Fig. 9) have the slopes $r_{1}, r_{2}$ similar to slopes obtained in the numerical calculations and shown in Fig. 6 (see also Fig. 10 in papers by Imada et al., 2007). Observed energies of the spectrum knee for majority of cases are $\varepsilon^{*} \sim 100$ $150 \mathrm{keV}$. If $\varepsilon_{0} \sim 1 \mathrm{keV}$, then according to the presented model we have $\varepsilon^{*} / \varepsilon_{0} \sim 30-300$. To obtain $\varepsilon^{*} \sim 100-150 \mathrm{keV}$ we need to take $L_{\mathrm{z}} \approx \rho_{0 \mathrm{p}} \approx 10^{3} \mathrm{~km}, L_{\mathrm{x}} \approx 10 \rho_{\mathrm{p}} \approx 10^{4} \mathrm{~km}, E_{\mathrm{y}} \approx$ 
$5 \mathrm{mV} \mathrm{m}^{-1}$, and $E_{0} \approx 100 \mathrm{mV} \mathrm{m}^{-1}$. These are typical parameters for the magnetotail. Therefore, our model gives correct estimates of the spectrum knee energy.

Of course, there are several alternative mechanisms which can be responsible for electron acceleration in the magnetotail. First of all, rapid increase of $B_{\mathrm{Z}}$ component due to spontaneous current filament disruptions could be responsible for sharp bursts of the electric field in the vicinity of the neutral plane (i.e., small scale transient magnetic reconnections). Although such induction electric field accelerates electrons, the final energy distribution is exponential (see theoretical model and comparison with observations in Taktakishvili et al., 1998). Another possible candidate is lower-hybrid waves often observed in the vicinity of the X-line (see, e.g., Shinohara et al., 1998; Fujimoto et al., 2011). These waves can effectively accelerate electrons via resonant wave-particle interaction. Also more sophisticated mechanisms related to dynamics of magnetic islands (see, e.g., Drake et al., 2005; Oka et al., 2010; Tanaka et al., 2011) could be responsible for additional acceleration. Finally, it is unclear how electron holes observed near the X-line (see review by Fujimoto et al., 2011, and references therein) can influence electron acceleration. However, here we notice that neither wave-particle resonant interaction nor various transient processes related to the reconnection can produce observed double-power law spectra (see, e.g., Birn et al., 2004, 2012, and references therein) and should be considered only as additional effects for the acceleration in X-line.

Presence of the electrostatic fields $E_{\mathrm{X}}, E_{\mathrm{Z}}$ results in electron trapping in the vicinity of the $\mathrm{X}$-line. This effect is similar to the well known surfatron mechanism of charged particle acceleration by electrostatic waves (Sagdeev and Shapiro, 1973; Katsouleas and Dawson, 1983; Neishtadt et al., 1989; Itin et al., 2000), where electric field compensates Lorentz force $\boldsymbol{v} \times \boldsymbol{B}$, while particles gain energy due to acceleration along the wave-front. It is worth noting that the similar mechanism of Lorentz force compensation by strongly inhomogeneous electric field was considered by Cole (1976) and also was studied for electron heating in collisionless shocks (e.g., Galeev et al., 1988; Balikhin et al., 1993; Lembège et al., 2003, and references therein), where the surfatron mechanism is traditionally considered (Ucer and Shapiro, 2001; Hoshino and Shimada, 2002). For nonrelativistic particles the velocity $v_{\mathrm{y}}$ grows infinitely. Thus, after certain time the Lorentz force exceeds the force of the electrostatic field. Therefore, eventually particles should leave the trapping regime, like it was found for classical nonrelativistic surfatron (Sagdeev and Shapiro, 1973). The situation can be substantially different for relativistic particles: as was shown by Katsouleas and Dawson (1983) the natural limitation of the Lorentz force due to $|\boldsymbol{v} / c|<1$ can result in infinite trapping. The similar effect can be obtained in our system of electron motion in the vicinity of the X-line (see Appendix C). For particles with initially large energy the acceleration is more stable, but the infinite trapping is possible only in the unrealistic case $E_{0} / B_{0} \sim 1$. Although, this situation cannot be realised in the magnetotail, so intense electric fields could present in the relativistic reconnection in pulsar magnetospheres (see, e.g., Zenitani and Hoshino, 2007; Cerutti et al., 2012, and references therein), where trapping acceleration may be responsible for production of high-energy particles.

In the vicinity of the $\mathrm{X}$-line, various plasma instabilities can develop. This leads to the formation of electromagnetic field fluctuations (see review by Fujimoto et al., 2011, and references therein). The role of these fluctuations for particle acceleration and scattering is a subject of separate studies. However, here we would like to discuss the influence of highfrequency magnetic field fluctuations on stability of electron acceleration in the non-adiabatic region. For example, it was shown that in the case of classical surfatron acceleration the magnetic field fluctuations lead to particle escape from the trapped regime of acceleration (Artemyev et al., 2011b). For the X-line geometry our estimates demonstrate that fluctuations could substantially change particle dynamics only if the power spectra density of fluctuations exceeds certain level (see Appendix B). This level is higher than level of observed fluctuations. Thus, the trapped acceleration appears to be stable relative to this effect.

As opposed to nonrelativistic particle interaction with electrostatic waves (Sagdeev and Shapiro, 1973), where acceleration is limited by finite time of trapped motion, it was shown that the capture of nonrelativistic particle by electromagnetic waves is more stable and particle acceleration can not be stopped (Neishtadt et al., 2009; Artemyev et al., 2010). Analogous effect can be obtained for electron acceleration in the vicinity of the X-line, if $E_{\mathrm{X}}$ component corresponds to an induction electric field in the developing $X$-line with $\partial B_{\mathrm{Z}} / \partial t \neq 0$ (see Appendix D). During X-line growth, electrons are trapped and gain energy due to direct acceleration by $E_{\mathrm{y}}$ component. We notice, that explosive growth of the Xline provides stable acceleration of relativistic electrons even in the system without $E_{\mathrm{x}}, E_{\mathrm{Z}}$ components (Birn et al., 2012). However, both these mechanisms require time scales of the $\mathrm{X}$-line dynamics comparable with the time scale of electron motion. We also mention that additional acceleration by induction electric field can mimic the effect of adiabatic heating corresponding to increase of magnetic field amplitude in the vicinity of the $\mathrm{X}$-line. Such an increase is possible in case of strong external driving of the magnetic reconnection (Hoshino, 2005; Birn et al., 2005, and references therein) or due to initial accumulation of magnetic flux in the magnetotail current sheet (Sitnov and Swisdak, 2011).

In this paper, we did not discuss the role of the guide field. However, this component of magnetic field (if exists) is extremely important for particle dynamics, because it can magnetise electrons even in the X-line and support their stable acceleration (Bulanov, 1980; Browning and Vekstein, 2001; Egedal et al., 2008). However, the influence of the guide field on surfatron trapped acceleration is still an open question. Here we can only mention from Fig. 9, that the knee energy 
of event (b) is lower than for other two events (a) and (c), and the event (b) seems to have larger $B_{\mathrm{y}}$ component. Therefore, our observations point out to possible decrease of efficiency of surfatron acceleration with the increase of strength of the guide field. Analytical description of formation of energy distribution in the system with nonzero guide component is a substantially more complicated task and we leave it for further work.

We should notice that presented theory is able to explain only the value of energy of the spectrum knee, $\varepsilon^{*}$, while we did not obtain estimates for slopes $r_{1}, r_{2}$ of two spectrum fragments. Analytical estimates of these slope values seem to be important for comparison with experimental observations. Such estimates may be obtained by utilising the approach introduced by Bulanov and Sasorov (1976); Galeev (1979); Zelenyi et al. (1990a). We left this piece of work for further publications.

\section{Conclusions}

In this paper, we develop the analytical model of electron acceleration in the vicinity of the X-line based on concept of surfing acceleration mechanism proposed by Hoshino (2005). We show that accelerated electrons form energy spectra with typical double power-law structure and estimate the energy of the spectrum knee $\varepsilon^{*}$. For typical conditions of the Earth magnetotail we obtain $\varepsilon^{*} \sim 100-150 \mathrm{keV}$. Our model predicts more stable acceleration for electrons with larger initial energies.

We select several events of spacecraft (Interball and Geotail) observations of the double power-law spectra in the vicinity of the $\mathrm{X}$-line in the magnetotail. Our comparison of theory with spacecraft observation demonstrates that the model can reproduce their main features.

\section{Appendix A}

\section{Test particle approach}

We present electrons trajectories and energy distributions obtained by numerical integration of Hamiltonian equations of motion. The following configuration of magnetic and electric fields is used for integration of trajectories:

$$
\left\{\begin{array}{l}
B_{\mathrm{x}} / B_{0}=\alpha_{\mathrm{z}} z,|z|<\alpha_{\mathrm{z}}^{-1},|x|<\alpha_{\mathrm{x}}^{-1} \\
B_{\mathrm{z}} / B_{0}=\alpha_{\mathrm{x}} x,|z|<\alpha_{\mathrm{z}}^{-1},|x|<\alpha_{\mathrm{x}}^{-1} \\
c E_{\mathrm{y}} / \rho_{0} \omega_{0} B_{0}=v_{\mathrm{D}},|z|<\alpha_{\mathrm{z}}^{-1},|x|<\alpha_{\mathrm{x}}^{-1} \\
c E_{0} / \rho_{0} \omega_{0} B_{0}=w_{\mathrm{D}},|z|<\ell_{\mathrm{z}},|x|<\ell_{\mathrm{x}}
\end{array}\right.
$$

We suppose that magnetic field is stationary (i.e., $\partial \boldsymbol{B} / \partial t=$ $0)$. Thus, $E_{\mathrm{x}}$ and $E_{\mathrm{z}}$ are components of electrostatic field. These components are not equal to zero only inside the domain of non-adiabatic electron motion $|x|<\ell_{\mathrm{x}},|z|<\ell_{\mathrm{z}}$. Electric field $E_{\mathrm{y}}$ is present in the whole domain of modelling.
In order to obtain the energy distribution, we run $1.5 \times 10^{6}$ particles starting from $x=0, z=0$ point with randomly distributed initial velocities. Initial energy is distributed accordingly to the Maxwell function with temperature $\varepsilon_{0}$.

\section{Appendix B}

\section{Stability of acceleration}

In this Appendix, we consider stability of the particle acceleration in the domain of non-adiabatic motion. We suppose that high-frequency fluctuations $\delta B_{\mathrm{Z}}$ of the magnetic field exist in the system:

$\dot{v}_{\mathrm{x}}=-\alpha_{\mathrm{x}} \sigma x\left(w_{\mathrm{D}}-t v_{\mathrm{D}}\right)+t v_{\mathrm{D}} \delta b_{\mathrm{z}}$

Here we use the normalisation $\delta b_{\mathrm{z}}=\delta B_{\mathrm{z}} / B_{0}$. Frequency of fluctuations is $\omega$ and the dimensionless period $\tau=\omega_{0} / \omega$ is much smaller than the typical time scale of particle dynamics $\sim\left(v_{\mathrm{D}} \alpha_{\mathrm{x}} \sigma\right)^{-1 / 3}$. In this case, we can separate the time scales and write the solution of Eq. (B1) as a sum $v_{\mathrm{x}}=v_{\mathrm{x}}^{(0)}+v_{\mathrm{x}}^{(\delta)}$, where $v_{\mathrm{x}}^{(0)}$ is solution for the system without $\delta b_{\mathrm{z}}$. Velocity fluctuations for a given moment $t_{i}=i \tau$ can be written as $\Delta v_{\mathrm{x}}^{(\delta)}\left(t_{i}\right)=t_{i} v_{\mathrm{D}} \delta b_{\mathrm{z}}\left(t_{i}\right) \tau$. Assuming statistical independence of fluctuations (i.e., $\left\langle\delta b_{\mathrm{z}}\left(t_{\mathrm{i}}\right) \delta b_{\mathrm{z}}\left(t_{j}\right)\right\rangle=0$ if $i \neq j$ ) one can write the expression for velocity fluctuations:

$v_{\mathrm{x}}^{(\delta)} \sim \sqrt{\sum_{i}\left(\Delta v_{\mathrm{x}}^{(\delta)}\left(t_{i}\right)\right)^{2}} \sim t^{3 / 2} v_{\mathrm{D}} \sqrt{\sigma_{\mathrm{z}} \tau}$

where $\sigma_{\mathrm{z}}=\left\langle\delta b_{\mathrm{z}}^{2}\left(t_{i}\right)\right\rangle$ is the variance of $\delta b_{\mathrm{z}}$ distribution. After integration we obtain corresponding evolution of coordinate:

$x^{(\delta)} \sim v_{\mathrm{D}} t^{5 / 2} \sqrt{\sigma_{\mathrm{z}} \tau}$

Therefore, electrons reach the boundary $x \sim \ell_{\mathrm{x}}$ in time period $T^{(\delta)} \sim \ell_{\mathrm{x}}^{2 / 5} v_{\mathrm{D}}^{-2 / 5}\left(\sigma_{\mathrm{z}} \tau\right)^{-1 / 5}$. If $T^{(\delta)}<T^{\text {nad }} \sim$ $\ell_{\mathrm{x}}^{1 / 4}\left(\alpha_{\mathrm{X}} \sigma v_{\mathrm{D}}\right)^{-1 / 3}$ then the influence of magnetic field fluctuations can substantially reduce time of particle acceleration. This inequality can be rewritten as:

$\sigma_{\mathrm{z}} \tau>\alpha_{\mathrm{x}}^{31 / 12} \alpha_{\mathrm{z}}^{-1 / 6} v_{\mathrm{D}}^{-1 / 3} \sim 10^{-6}$

In dimensional form, we can write $\delta B_{\mathrm{z}}^{2} / \omega>1 \mathrm{nT}^{2} \mathrm{~Hz}^{-1}$ for $\omega>100 \mathrm{~Hz}$, where we assume $B_{0} \sim 10 \mathrm{nT}$. Fluctuations of magnetic field with so strong power spectral density are not observed (see, e.g., Hoshino et al., 1994; Petrukovich, 2005; Fujimoto et al., 2011, and references therein). Therefore, we can conclude that small-scale fluctuations of magnetic field cannot stop particle acceleration in the domain of nonadiabatic motion. 


\section{Appendix C}

\section{Relativistic regime}

This Appendix is devoted to the consideration of the relativistic electron motion in the vicinity of the X-line. Hamiltonian equations of motion in this case are

$\left\{\begin{array}{l}\dot{p}_{\mathrm{x}} / m_{\mathrm{e}}=-\omega_{0} \sigma v_{\mathrm{y}}\left(x / L_{\mathrm{x}}\right)-\omega_{0} \sigma w_{\mathrm{D}}\left(x / L_{\mathrm{x}}\right) \\ \dot{p}_{\mathrm{y}} / m_{\mathrm{e}}=-\omega_{0} v_{\mathrm{D}}-\omega_{0}\left(v_{\mathrm{z}}\left(z / L_{\mathrm{z}}\right)-v_{\mathrm{x}} \sigma\left(x / L_{\mathrm{x}}\right)\right) \\ \dot{p}_{\mathrm{z}} / m_{\mathrm{e}}=\omega_{0} v_{\mathrm{y}}\left(z / L_{\mathrm{z}}\right)+\omega_{0} w_{\mathrm{D}}\left(z / L_{\mathrm{z}}\right)\end{array}\right.$

where $\left\{p_{\mathrm{x}}, p_{\mathrm{y}}, p_{\mathrm{z}}\right\}$ are components of electron momentum. We introduce dimensionless variables: $\left\{v_{\mathrm{x}}, v_{\mathrm{y}}, v_{\mathrm{z}}\right\} / c \rightarrow\left\{v_{\mathrm{x}}, v_{\mathrm{y}}, v_{\mathrm{z}}\right\} / c, \quad\left\{p_{\mathrm{x}}, p_{\mathrm{y}}, p_{\mathrm{z}}\right\} / m_{\mathrm{e}} c \rightarrow$ $\left\{p_{\mathrm{x}}, p_{\mathrm{y}}, p_{\mathrm{z}}\right\}, \quad\{x, y, z\} \omega_{0} / c \rightarrow\{x, y, z\}, \quad \beta_{\mathrm{x}, \mathrm{z}}=c / L_{\mathrm{x}, \mathrm{z}} \omega_{0}$, $t \omega \rightarrow t, \quad v_{\mathrm{D}}, w_{\mathrm{D}} / c \rightarrow v_{\mathrm{D}}, w_{\mathrm{D}}$, and dimensionless energy $\gamma=\sqrt{1+p_{\mathrm{x}}^{2}+p_{\mathrm{y}}^{2}+p_{\mathrm{z}}^{2}}$. Considering particle motion in the vicinity of $\mathrm{X}$-line we obtain $v_{\mathrm{y}}=p_{\mathrm{y}} / \gamma \approx-t v_{\mathrm{D}} / \gamma$. Then we can rewrite system of equations

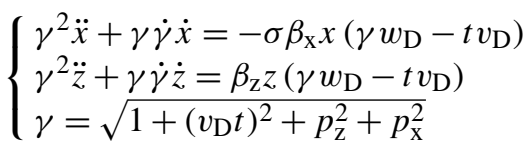

For high energy particles $\left(\gamma \sim v_{\mathrm{D}} t\right)$ we obtain

$\left\{\begin{array}{l}t \ddot{x}+\dot{x}=-\frac{\sigma \beta_{\mathrm{X}}}{v_{\mathrm{D}}} x\left(w_{\mathrm{D}}-1\right) \\ t \ddot{z}+\dot{z}=\frac{\beta_{\mathrm{z}}}{v_{\mathrm{D}}} z\left(w_{\mathrm{D}}-1\right)\end{array}\right.$

Solution of this system is

$$
\begin{aligned}
& x(t)=\text { const } \cdot I_{0}\left(2 \sqrt{\frac{\sigma \beta_{\mathrm{X}}}{v_{\mathrm{D}}}\left(1-w_{\mathrm{D}}\right) t}\right) \\
& z(t)=\text { const } \cdot J_{0}\left(2 \sqrt{\frac{\beta_{\mathrm{Z}}}{v_{\mathrm{D}}}\left(1-w_{\mathrm{D}}\right) t}\right)
\end{aligned}
$$

Here $I_{0}, J_{0}$ are Bessel functions of imaginary and real arguments, respectively. The absolute value of $x$ coordinate increases exponentially with time. Thus, electrons can spend in the vicinity of the X-line only time interval $T^{\text {nad }} \approx\left(\sigma \beta_{\mathrm{x}}\right)^{-1} v_{\mathrm{D}} /\left(1-w_{\mathrm{D}}\right)$. Corresponding energy gain is $\gamma \sim v_{\mathrm{D}} T^{\text {nad }}=\left(\sigma \beta_{\mathrm{x}}\right)^{-1} v_{\mathrm{D}}^{2} /\left(1-w_{\mathrm{D}}\right)$. Therefore, electrons can gain substantial energy if $1-w_{\mathrm{D}}$ is close to zero.

To check dependence $\gamma \sim 1 /\left(1-w_{\mathrm{D}}\right)$ we integrate numerically three trajectories with various $w_{\mathrm{D}}$. Figure $\mathrm{C} 1$ shows that increase of $w_{\mathrm{D}}$ parameter leads to increase of energy gain $\gamma$ inside the domain $|x|<\ell_{\mathrm{x}}$ in agreement with the scaling $\gamma \sim 1 /\left(1-w_{\mathrm{D}}\right)$.

\section{Appendix D}

\section{Effect of induction field}

We consider the system with evolution of $B_{\mathrm{Z}}=\sigma B_{0} \alpha_{\mathrm{x}}(t) x$ component and corresponding component of induction electric field $E_{\mathrm{x}}=\left(\sigma B_{0} \rho_{0} \omega_{0} / c\right) \dot{\alpha}_{\mathrm{x}}(t) x y$. Equation of particle
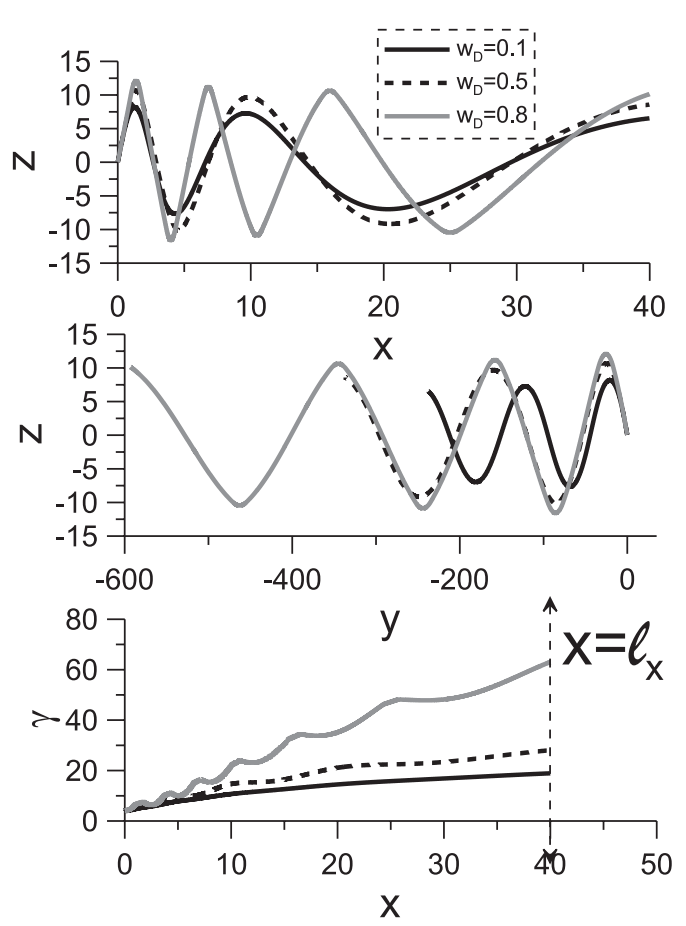

Fig. C1. Three particle trajectories and energy as a function of $x$ coordinate are shown for various $w_{\mathrm{D}}$. We use $v_{\mathrm{D}}=0.01, \beta_{\mathrm{X}}=10^{-3}$ and $\beta_{\mathrm{Z}}=10^{-2}$.

motion along $\mathrm{x}$-direction in this case has the form:

$\dot{v}_{\mathrm{x}}=-\sigma x\left(\alpha_{\mathrm{x}} v_{\mathrm{y}}+\dot{\alpha}_{\mathrm{x}} y\right)$

where $v_{\mathrm{y}} \approx-v_{\mathrm{D}} t$ and $y \approx-(1 / 2) v_{\mathrm{D}} t^{2}$. Substituting expressions for $v_{\mathrm{y}}$ and $y$ into the equation of motion, we obtain:

$\dot{v}_{\mathrm{x}}=\sigma x v_{\mathrm{D}} t\left(\alpha_{\mathrm{x}}+\frac{1}{2} \dot{\alpha}_{\mathrm{x}} t\right)$

Then if $\alpha_{\mathrm{x}} \sim t^{-n}$ and $n>2$ we have $\ddot{x}=-\sigma x v_{\mathrm{D}} t^{1-n}(n-$ 2) $/ 2$ and motion along $x$-direction is stable, i.e., $|x(t)|<$ const for all $t$. The dependence $\alpha_{\mathrm{x}} \sim t^{-n}$ corresponds to the expansion of the $\mathrm{X}$-line ( $L_{\mathrm{x}}$ grows).

Acknowledgements. We are very grateful to both reviewers for useful comments and suggestions. A.V.A. is thankful to V. V. Krasnoselskikh for fruitful discussions related to the mechanism of electron demagnetisation in the shock waves. Authors would like to acknowledge Interball-tail and Geotail instrument teams ASPI/MIFM, CORALL, ELECTRON, DOK-2, FGM, LEP and EPIC for excellent data. Work of A.V.A., A.A.P., L.M.Z. was supported by the Russian Foundation for Basic Research (projects no. 12-02-31127, 10-02-00135) and by the European Union Seventh Framework Programme [FP7/2007-2013] under grant agreement no. 269198 Geoplasmas (Marie Curie International Research Staff Exchange Scheme).

Topical Editor R. Nakamura thanks A. Anastasiadis and one anonymous referee for their help in evaluating this paper. 


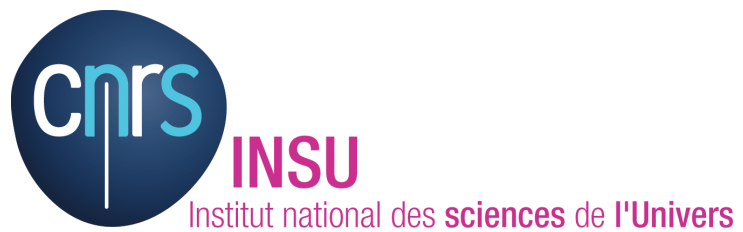

The publication of this article is financed by CNRS-INSU.

\section{References}

Anastasiadis, A., Gontikakis, C., and Efthymiopoulos, C.: Particle Interactions with Single or Multiple 3D Solar Reconnecting Current Sheets, Solar Physics, 253, 199-214, doi:10.1007/s11207008-9234-4, 2008.

Artemyev, A. V., Neishtadt, A. I., Zelenyi, L. M., and Vainchtein, D. L.: Adiabatic description of capture into resonance and surfatron acceleration of charged particles by electromagnetic waves, Chaos, 20, 043128, doi:10.1063/1.3518360, 2010.

Artemyev, A. V., Baumjohann, W., Petrukovich, A. A., Nakamura, R., Dandouras, I., and Fazakerley, A.: Proton/electron temperature ratio in the magnetotail, Ann. Geophys., 29, 2253-2257, doi:10.5194/angeo-29-2253-2011, 2011a.

Artemyev, A., Vainchtein, D., Neishtadt, A., and Zelenyi, L.: Resonant acceleration of charged particles in the presence of random fluctuations, Phys. Rev. E, 84, 046213, doi:10.1103/PhysRevE.84.046213, 2011 b.

Artemyev, A. V., Lutsenko, V. N., and Petrukovich, A. A.: Ion resonance acceleration by dipolarization fronts: analytic theory and spacecraft observation, Ann. Geophys., 30, 317-324, doi:10.5194/angeo-30-317-2012, 2012.

Asano, Y., Shinohara, I., Retinò, A., Daly, P. W., Kronberg, E. A., Takada, T., Nakamura, R., Khotyaintsev, Y. V., Vaivads, A., Nagai, T., Baumjohann, W., Fazakerley, A. N., Owen, C. J., Miyashita, Y., Lucek, E. A., and Rème, H.: Electron acceleration signatures in the magnetotail associated with substorms, J. Geophys. Res., 115, A05215, doi:10.1029/2009JA014587, 2010.

Ashour-Abdalla, M., Berchem, J. P., Büchner, J., and Zelenyi, L. M.: Shaping of the magnetotail from the mantle - Global and local structuring, J. Geophys. Res., 98, 5651-5676, doi:10.1029/92JA01662, 1993.

Ashour-Abdalla, M., Zelenyi, L. M., Peroomian, V., and Richard, R. L.: Consequences of magnetotail ion dynamics, J. Geophys. Res., 99, 14891-14916, doi:10.1029/94JA00141, 1994.

Ashour-Abdalla, M., Zelenyi, L. M., Peroomian, V., Richard, R. L., and Bosqued, J. M.: The mosaic structure of plasma bulk flows in the Earth's magnetotail, J. Geophys. Res., 100, 19191-19210, doi:10.1029/95JA00902, 1995.

Balikhin, M., Gedalin, M., and Petrukovich, A.: New mechanism for electron heating in shocks, Phys. Rev. Lett., 70, 1259-1262, doi:10.1103/PhysRevLett.70.1259, 1993.

Birn, J. and Drake, J. F., Shay, M. A., Rogers, B. N., Denton, R. E., Hesse, M., Kuznetsova, M., Ma, Z. W., Bhattacharjee, A., Otto, A., and Pritchett, P. L.: Geospace Environmental Modeling (GEM) magnetic reconnection challenge, J. Geophys. Res., 106, 3715-3720, doi:10.1029/1999JA900449, 2001.

Birn, J., Thomsen, M. F., and Hesse, M.: Electron acceleration in the dynamic magnetotail: Test particle orbits in three-dimensional magnetohydrodynamic simulation fields, Phys. Plasmas, 11, 1825-1833, doi:10.1063/1.1704641, 2004.

Birn, J., Galsgaard, K., Hesse, M., Hoshino, M., Huba, J., Lapenta, G., Pritchett, P. L., Schindler, K., Yin, L., Büchner, J., Neukirch, T., and Priest, E. R.: Forced magnetic reconnection, Geophys. Res. Lett., 32, L06105, doi:10.1029/2004GL022058, 2005.

Birn, J., Artemyev, A. V., Baker, D. N., Echim, M., Hoshino, M., and Zelenyi, L. M.: Particle acceleration in the magnetotail and aurora, Space Sci. Rev., 173, 49-102, doi:10.1007/s11214-0129874-4, 2012.

Browning, P. K. and Vekstein, G. E.: Particle acceleration at an Xtype reconnection site with a parallel magnetic field, J. Geophys. Res., 106, 18677-18692, doi:10.1029/2001JA900014, 2001.

Büchner, J. and Zelenyi, L. M.: Deterministic chaos in the dynamics of charged particles near a magnetic field reversal, Phys. Lett. A, 118, 395-399, doi:10.1016/0375-9601(86)90268-9, 1986.

Büchner, J. and Zelenyi, L. M.: Regular and chaotic charged particle motion in magnetotaillike field reversals. I - Basic theory of trapped motion, J. Geophys. Res., 94, 11821-11842, doi:10.1029/JA094iA09p11821, 1989.

Bulanov, S. V.: The energy spectrum of particles accelerated near a singular magnetic field line, Soviet Astronomy Letters, 6, 206, 1980.

Bulanov, S. V. and Sasorov, P. V.: Energy spectrum of particles accelerated in the neighborhood of a line of zero magnetic field, Soviet Astronomy, 19, 464-468, 1976.

Burkhart, G. R., Drake, J. F., and Chen, J.: Magnetic reconnection in collisionless plasmas - Prescribed fields, J. Geophys. Res., 95, 18833-18848, doi:10.1029/JA095iA11p18833, 1990.

Cerutti, B., Uzdensky, D. A., and Begelman, M. C.: Extreme Particle Acceleration in Magnetic Reconnection Layers: Application to the Gamma-Ray Flares in the Crab Nebula, Astrophys. J. , 746, 148, doi:10.1088/0004-637X/746/2/148, 2012.

Chen, J.: Nonlinear dynamics of charged particles in the magnetotail, J. Geophys. Res., 97, 15011, doi:10.1029/92JA00955, 1992.

Cole, K. D.: Effects of crossed magnetic and spatially dependent electric fields on charged particle motion, Plan. Sp. Sci., 24, 515-518, doi:10.1016/0032-0633(76)90096-9, 1976.

Cowley, S. W. H. and Shull Jr., P.: Current sheet acceleration of ions in the geomagnetic tail and the properties of ion bursts observed at the lunar distance, Planet. Space Sci., 31, 235-245, doi:10.1016/0032-0633(83)90058-2, 1983.

Divin, A., Markidis, S., Lapenta, G., Semenov, V. S., Erkaev, N. V., and Biernat, H. K.: Model of electron pressure anisotropy in the electron diffusion region of collisionless magnetic reconnection, Phys. Plasmas, 17, 122102, doi:10.1063/1.3521576, 2010.

Divin, A., Lapenta, G., Markidis, S., Semenov, V. S., Erkaev, N. V., Korovinskiy, D. B., and Biernat, H. K.: Scaling of the inner electron diffusion region in collisionless magnetic reconnection, J. Geophys. Res., 117, A06217, doi:10.1029/2011JA017464, 2012.

Dobrowolny, M.: Instability of a neutral sheet, Nuovo Cimento B Series, 55, 427-442, doi:10.1007/BF02711653, 1968.

Drake, J. F., Shay, M. A., Thongthai, W., and Swisdak, M.: Production of Energetic Electrons during Magnetic Reconnection, Phys. Rev. Lett., 94, 095001, doi:10.1103/PhysRevLett.94.095001, 2005.

Drake, J. F., Swisdak, M., Che, H., and Shay, M. A.: Electron acceleration from contracting magnetic islands during reconnection, Nature, 443, 553-556, doi:10.1038/nature05116, 2006. 
Egedal, J., Fox, W., Katz, N., Porkolab, M., Øieroset, M., Lin, R. P., Daughton, W., and Drake, J. F.: Evidence and theory for trapped electrons in guide field magnetotail reconnection, J. Geophys. Res., 113, A12207, doi:10.1029/2008JA013520, 2008.

Fujimoto, M., Shinohara, I., and Kojima, H.: Reconnection and Waves: A Review with a Perspective, Space Sci. Rev., 160, 123 143, doi:10.1007/s11214-011-9807-7, 2011.

Galeev, A. A.: Reconnection in the magnetotail, Space Science Reviews, 23, 411-425, doi:10.1007/BF00172248, 1979.

Galeev, A. A., Krasnosel'skikh, V. V., and Lobzin, V. V.: Fine structure of the front of a quasi-perpendicular supercritical collisionless shock wave, Soviet Journal of Plasma Physics, 14, 1192 1200, 1988.

Hannah, I. G. and Fletcher, L.: Comparison of the Energy Spectra and Number Fluxes From a simple Flare Model to Observations, Solar Physics, 236, 59-74, doi:10.1007/s11207-006-01399, 2006

Hesse, M., Birn, J., and Kuznetsova, M.: Collisionless magnetic reconnection: Electron processes and transport modeling, J. Geophys. Res., 106, 3721-3736, doi:10.1029/1999JA001002, 2001.

Hoshino, M.: Electron surfing acceleration in magnetic reconnection, J. Geophys. Res., 110, 10215, doi:10.1029/2005JA011229, 2005.

Hoshino, M. and Shimada, N.: Nonthermal Electrons at High Mach Number Shocks: Electron Shock Surfing Acceleration, Astrophys. J. , 572, 880-887, doi:10.1086/340454, 2002.

Hoshino, M., Nishida, A., Yamamoto, T., and Kokubun, S.: Turbulent magnetic field in the distant magnetotail: Bottom-up process of plasmoid formation?, Geophys. Res. Lett., 21, 2935-2938, doi:10.1029/94GL02094, 1994.

Imada, S., Nakamura, R., Daly, P. W., Hoshino, M., Baumjohann, W., Mühlbachler, S., Balogh, A., and Rème, H.: Energetic electron acceleration in the downstream reconnection outflow region, J. Geophys. Res., 112, A03202, doi:10.1029/2006JA011847, 2007.

Imada, S., Hirai, M., Hoshino, M., and Mukai, T.: Favorable conditions for energetic electron acceleration during magnetic reconnection in the Earth's magnetotail, J. Geophys. Res., 116, A08217, doi:10.1029/2011JA016576, 2011.

Itin, A. P., Neishtadt, A. I., and Vasiliev, A. A.: Captures into resonance and scattering on resonance in dynamics of a charged relativistic particle in magnetic field and electrostatic wave, Physica D: Nonlinear Phenomena, 141, 281-296, doi:10.1016/S01672789(00)00039-7, 2000.

Karimabadi, H., Daughton, W., and Scudder, J.: Multi-scale structure of the electron diffusion region, Geophys. Res. Lett., 34, 13104, doi:10.1029/2007GL030306, 2007.

Katsouleas, T. and Dawson, J. M.: Unlimited electron acceleration in laser-driven plasma waves, Phys. Rev. Lett., 51, 392-395, doi:10.1103/PhysRevLett.51.846.2, 1983.

Klimov, S., Romanov, S., Amata, E., Blecki, J., Büchner, J., Juchniewicz, J., Rustenbach, J., Triska, P., Woolliscroft, L. J. C., Savin, S., Afanas'yev, Yu., de Angelis, U., Auster, U., Bellucci, G., Best, A., Farnik, F., Formisano, V., Gough, P., Grard, R., Grushin, V., Haerendel, G., Ivchenko, V., Korepanov, V., Lehmann, H., Nikutowski, B., Nozdrachev, M., Orsini, S., Parrot, M., Petrukovich, A., Rauch, J. L., Sauer, K., Skalsky, A., Slominski, J., Trotignon, J. G., Vojta, J., and Wronowski, R.: ASPI experiment: measurements of fields and waves on board the INTERBALL-1 spacecraft, Ann. Geophys., 15, 514-527, doi:10.1007/s00585-997-0514-3, 1997.

Kokubun, S., Yamamoto, T., Acuna, M. H., Hayashi, K., Shiokawa, K., and Kawano, H.: The GEOTAIL magnetic field experiment, J. Geomagn. Geoelectr., 46, 7-21, 1994.

Korovinskiy, D. B., Semenov, V. S., Erkaev, N. V., Divin, A. V., and Biernat, H. K.: The 2.5-D analytical model of steadystate Hall magnetic reconnection, J. Geophys. Res., 113, 4205, doi:10.1029/2007JA012852, 2008.

Lembège, B. and Pellat, R.: Stability of a thick twodimensional quasineutral sheet, Phys. Fl., 25, 1995-2004, doi:10.1063/1.863677, 1982.

Lembège, B., Savoini, P., Balikhin, M., Walker, S., and Krasnoselskikh, V.: Demagnetization of transmitted electrons through a quasi-perpendicular collisionless shock, J. Geophys. Res., 108, 1256, doi:10.1029/2002JA009288, 2003.

Litvinenko, Y. E.: Three-dimensional fan magnetic reconnection and particle acceleration in the solar corona, Astronomy and Astrophysics, 452, 1069-1074, doi:10.1051/0004-6361:20054324, 2006.

Lutsenko, V. N. and Kudela, K.: Almost monoenergetic ions near the Earth's magnetosphere boundaries, Geophys. Res. Lett., 26 , 413-416, doi:10.1029/1999GL900002, 1999.

Lutsenko, V. N., Kudela, K., and Sarris, E. T.: The DOK-2 Experiment to Study Energetic Particles by the Tail Probe and Auroral Probe Satellites in the INTERBALL Project, Cosmic Research, 36, 98-107, 1998.

Lyons, L. R.: Electron energization in the geomagnetic tail current sheet, J. Geophys. Res., 89, 5479-5487, doi:10.1029/JA089iA07p05479, 1984.

Lyons, L. R. and Speiser, T. W.: Evidence for current sheet acceleration in the geomagnetic tail, J. Geophys. Res., 87, 2276-2286, doi:10.1029/JA087iA04p02276, 1982.

Moses, R. W., Finn, J. M., and Ling, K. M.: Plasma heating by collisionless magnetic reconnection - Analysis and computation, J. Geophys. Res., 98, 4013-4040, 1993.

Mukai, T., Machida, S., Saito, Y., Hirahara, M., Terasawa, T., Kaya, N., Obara, T., Ejiri, M., and Nishida, A.: The low energy particles (LEP) experiment onboard the GEOTAIL satellite, J. Geomagn. Geoelectr., 46, 669-692, 1994.

Neishtadt, A. I., Petrovichev, B. A., and Chernikov, A. A.: Particle entrainment into unlimited acceleration, Soviet Journal of Plasma Physics, 15, 1021-1023, 1989.

Neishtadt, A. I., Artemyev, A. V., Zelenyi, L. M., and Vainshtein, D. L.: Surfatron acceleration in electromagnetic waves with a low phase velocity, JETP Letters, 89, 441-447, doi:10.1134/S0021364009090045, 2009.

Oka, M., Fujimoto, M., Shinohara, I., and Phan, T. D.: Island surfing mechanism of electron acceleration during magnetic reconnection, J. Geophys. Res., 115, A08223, doi:10.1029/2010JA015392, 2010.

Petrukovich, A. A.: Low Frequency Magnetic Fluctuations in the Earth's Plasma Sheet, in: Astrophysics and Space Science Library, edited by: Sharma, A. S. and Kaw, P. K., vol. 321 of Astrophysics and Space Science Library, pp. 145, 2005.

Petrukovich, A. A., Sergeev, V. A., Zelenyi, L. M., Mukai, T., Yamamoto, T., Kokubun, S., Shiokawa, K., Deehr, C. S., Budnick, E. Y., Büchner, J., Fedorov, A. O., Grigorieva, V. P., Hughes, T. J., Pissarenko, N. F., Romanov, S. A., and Sandahl, I.: Two 
spacecraft observations of a reconnection pulse during an auroral breakup, J. Geophys. Res., 103, 47-60, doi:10.1029/97JA02296, 1998.

Pritchett, P. L.: Relativistic electron production during guide field magnetic reconnection, J. Geophys. Res., 111, 10212, doi:10.1029/2006JA011793, 2006.

Retinò, A., Nakamura, R., Vaivads, A., Khotyaintsev, Y., Hayakawa, T., Tanaka, K., Kasahara, S., Fujimoto, M., Shinohara, I., Eastwood, J. P., André, M., Baumjohann, W., Daly, P. W., Kronberg, E. A., and Cornilleau-Wehrlin, N.: Cluster observations of energetic electrons and electromagnetic fields within a reconnecting thin current sheet in the Earth's magnetotail, J. Geophys. Res., 113, A12215, doi:10.1029/2008JA013511, 2008.

Sagdeev, R. Z. and Shapiro, V. D.: Influence of transverse magnetic field on Landau damping, Soviet JETP Letters, 17, 279282,1973

Sauvaud, J.-A., Koperski, P., Beutier, T., Barthe, H., Aoustin, C., Thocaven, J. J., Rouzaud, J., Penou, E., Vaisberg, O., and Borodkova, N.: The INTERBALL-Tail ELECTRON experiment: initial results on the low-latitude boundary layer of the dawn magnetosphere, Ann. Geophys., 15, 587-595, doi:10.1007/s00585-9970587-z, 1997.

Shabansky, V. P.: Some Processes in the Magnetosphere, Space Sci. Rev., 12, 299-418, doi:10.1007/BF00165511, 1971.

Shinohara, I., Nagai, T., Fujimoto, M., Terasawa, T., Mukai, T., Tsuruda, K., and Yamamoto, T.: Low-frequency electromagnetic turbulence observed near the substorm onset site, J. Geophys. Res., 103, 20365-20388, doi:10.1029/98JA01104, 1998.

Sitnov, M. I. and Swisdak, M.: Onset of collisionless magnetic reconnection in two-dimensional current sheets and formation of dipolarization fronts, J. Geophys. Res., 116, A12216, doi:10.1029/2011JA016920, 2011.

Sonnerup, B. U. Ö.: Adiabatic particle orbits in a magnetic null sheet., J. Geophys. Res., 76, 8211-8222, doi:10.1029/JA076i034p08211, 1971.

Speiser, T. W.: Particle Trajectories in Model Current Sheets, 1, Analytical Solutions, J. Geophys. Res., 70, 4219-4226, doi:10.1029/JZ070i017p04219, 1965.

Speiser, T. W.: Particle Trajectories in Model Current Sheets, 2, Applications to Auroras Using a Geomagnetic Tail Model, J. Geophys. Res., 72, 3919-3932, doi:10.1029/JZ072i015p03919, 1967.

Taktakishvili, A. L., Zelenyi, L. M., Lutsenko, V. N., and Kudela, K.: On the Spectra of Energetic Particles in the Earth's Magnetotail, Cosmic Research, 36, 265-273, 1998.

Tanaka, K. G., Fujimoto, M., Badman, S. V., and Shinohara, I.: Dynamic magnetic island coalescence and associated electron acceleration, Phys. Plasmas, 18, 022903, doi:10.1063/1.3554660, 2011.
Tverskoy, B. A.: Main mechanisms in the formation of the Earth's radiation belts., Rev. Geophys. Space Phys., 7, 219-231, doi:10.1029/RG007i001p00219, 1969.

Ucer, D. and Shapiro, V. D.: Unlimited Relativistic Shock Surfing Acceleration, Phys. Rev. Lett., 87, 075001, doi:10.1103/PhysRevLett.87.075001, 2001.

Vaivads, A., Retinò, A., Khotyaintsev, Yu. V., and André, M.: Suprathermal electron acceleration during reconnection onset in the magnetotail, Ann. Geophys., 29, 1917-1925, doi:10.5194/angeo-29-1917-2011, 2011.

Vekstein, G. E. and Priest, E. R.: Nonlinear magnetic reconnection with collisionless dissipation, Phys. Plasmas, 2, 3169-3178, doi:10.1063/1.871149, 1995.

Williams, D. J., McEntire, R. W., Schlemm, C., Lui, A. T. Y., G., G., Christon, S. P., and Gliem, F.: Geotail Energetic Particles and Ion Composition Instrument, J. Geomag. Geoelect., 46, 39-57, 1994.

Wygant, J. R., Cattell, C. A., Lysak, R., Song, Y., Dombeck, J., McFadden, J., Mozer, F. S., Carlson, C. W., Parks, G., Lucek, E. A., Balogh, A., Andre, M., Reme, H., Hesse, M., and Mouikis, C.: Cluster observations of an intense normal component of the electric field at a thin reconnecting current sheet in the tail and its role in the shock-like acceleration of the ion fluid into the separatrix region, J. Geophys. Res., 110, A09206, doi:10.1029/2004JA010708, 2005.

Yermolaev, Yu. I., Fedorov, A. O., Vaisberg, O. L., Balebanov, V. M., Obod, Yu. A., Jimenez, R., Fleites, J., Llera, L., and Omelchenko, A. N.: Ion distribution dynamics near the Earth's bow shock: first measurements with the 2D ion energy spectrometer CORALL on the INTERBALL/Tail-probe satellite, Ann. Geophys., 15, 533-541, doi:10.1007/s00585-997-0533-0, 1997.

Zelenyi, L. M., Lominadze, J. G., and Taktakishvili, A. L.: Generation of the energetic proton and electron bursts in planetary magnetotails, J. Geophys. Res., 95, 3883-3891, doi:10.1029/JA095iA04p03883, 1990a.

Zelenyi, L. M., Zogin, D. V., and Büchner, J.: Quasiadiabatic dynamics of charged particles in the tail of the magnetosphere, Cosmic Research, 28, 369-380, 1990b.

Zenitani, S. and Hoshino, M.: Particle Acceleration and Magnetic Dissipation in Relativistic Current Sheet of Pair Plasmas, Astrophys. J. , 670, 702-726, doi:10.1086/522226, 2007.

Zharkova, V. V., Arzner, K., Benz, A. O., Browning, P., Dauphin, C., Emslie, A. G., Fletcher, L., Kontar, E. P., Mann, G., Onofri, M., Petrosian, V., Turkmani, R., Vilmer, N., and Vlahos, L.: Recent Advances in Understanding Particle Acceleration Processes in Solar Flares, Space Sci. Rev., 159, 357-420, doi:10.1007/s11214-011-9803-y, 2011. 